\title{
On a shallow water model for the simulation of turbidity currents
}

\author{
T. Morales de Luna ${ }^{1}$, M. J. Castro Díaz ${ }^{2}$, C. Parés Madroñal ${ }^{3}$, E.D. Fernández Nieto ${ }^{4}$ \\ $1,2,3$ Análisis Matemático \\ ${ }^{4}$ Dpto. Matemática Aplicada I \\ Facultad de Ciencias - Univ. Málaga \\ Campus de Teatinos, $\mathrm{s} / \mathrm{n}$ \\ ETS Arquitectura - Univ. Sevilla \\ Avda. Reina Mercedes N. 2 \\ 41012-Sevilla, Spain \\ E-mail: ${ }^{1}$ morales@anamat.cie.uma.es, ${ }^{2}$ castro@anamat.cie.uma.es, ${ }^{3}$ pares@anamat.cie.uma.es, ${ }^{4}$ edofer@us.es
}

\begin{abstract}
We present a model for hyperpycnal plumes or turbidity currents that takes into account the interaction between the turbidity current and the bottom, considering deposition and erosion effects as well as solid transport of particles at the bed load due to the current. Water entrainment from the ambient water in which the turbidity current plunges is also considered. Motion of ambient water is neglected and the rigid lid assumption is considered. The model is obtained as a depth-average system of equations under the shallow water hypothesis describing the balance of fluid mass, sediment mass and mean flow. The character of the system is analyzed and numerical simulations are carried out using finite volume schemes and path-conservative Roe schemes.
\end{abstract}

Key words: Turbidity currents, Hyperbolic systems, Finite Volume Methods, Path-conservative schemes, Numerical modeling

AMS subject classifications: 74S10, 35L60, 35L65, 74G15

\section{Introduction}

When a river contains an elevated concentration of suspended sediment, to the extent that the river density is greater than that of the receiving water body, the river can plunge and create a hyperpycnal plume or turbidity current. This hyperpycnal plume can travel significant distances until it loses its identity by entraining surrounding ambient water and dropping its sediment load. A sketch of a turbid underflow is presented in Figure 1.

There is great interest in turbidity currents because of their profound impact on the morphology of the continental shelves and ocean basins of the world. It is commonly accepted that they are one of the potential processes through which sediments can be transferred to the deep sea environments. These bottom currents influence the sea bed morphology by depositing, eroding and dispersing large quantities of sediment particles. The resultant deposit often form porous layer of rocks which are potential sources of hydrocarbon. Therefore, understanding and predicting the geometry of these deposits is crucial for effectively exploring and exploiting these reservoirs.

An additional concern is the destructive effect that turbidity currents have on underwater structures, such as cables, pipelines and foundations.

Large-scale hyperpycnal flow or turbidity currents in the natural environment are difficult to monitor because of the unpredictable nature of the events. As a result, most of our knowledge about these flows is derived from small scale laboratory experiments like the ones described in [19], [11],[1], [17] and [16].

In this paper, we present a numerical model of hyperpycnal flow generated by the plunging of a river. It incorporates the interaction between the turbidity current and bottom, considering eroding and deposit effects as well as solid transport due to the velocity of the current. Here, the along-shore 


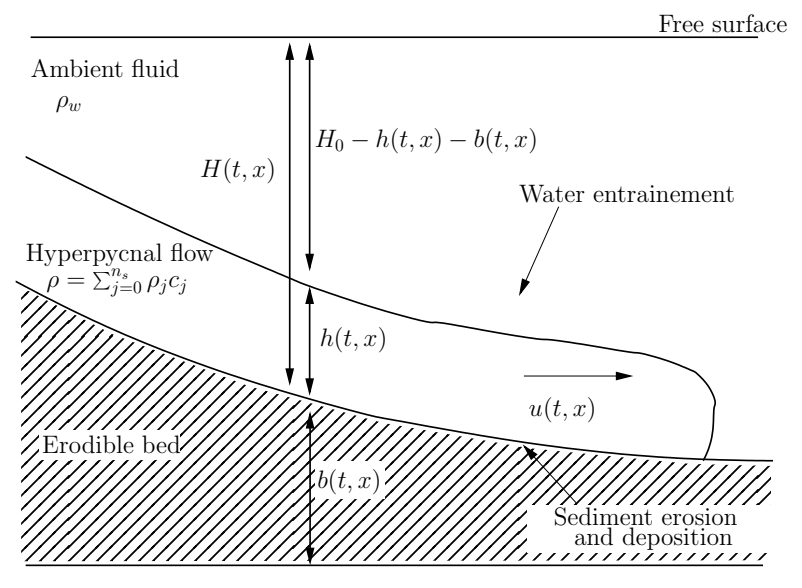

Figure 1: Sketch of hyperpycnal flow

current has been neglected. The equations are obtained as a layer-averaged system from the twodimensional equations under the shallow water hypothesis, namely the hydrostatic distribution of pressure.

A number of studies have attempted to predict the distribution of turbidities, mainly based on smallscale tank experiments of particle-driven density currents (see [8], [23], [15], [17], [16]). Numerical models have been developed as well, and some of them applied to the tank experiments (e.g. [3], [5], $[14])$.

The organization of this paper is as follows:

In Section 2 we describe the model presented here and in particular the empirical relations and physical constants taken into account.

In Section 3 the model is obtained from the two dimensional equations under the shallow water hypothesis and hydrostatic distribution of pressure.

The character of the system as well as its eigenstructure is studied in Section 4. An entropy inequality is also obtain under some hypothesis.

The theory presented in [22] and [21] is adapted in Section 5 in order to define a Roe scheme for the model considered here.

Finally, numerical results and comparisons to experimental data are shown in Section 7.

\section{Model description}

We introduce a layer-averaged model in which equations for conservation of fluid mass, sediment mass and fluid momentum are solved simultaneously. Vast applicability, low computational cost and conceptual simplicity are the main reasons that justify the option for layer averaged model in a onedimensional framework. This type of model has been widely used in the simulation of turbidity currents (e.g., [8], [23], [5], [17]) and some layer-averaged models have already been proposed in [23], [16], [17], [5] and [14]. In the framework proposed by these authors, $n_{s} \geq 1$ species of sediments are considered with constant density $\rho_{j}$, for $j=1, \ldots, n_{s}$. These sediment species are transported by a river with freshwater of constant density $\rho_{0}$. The river plunges into an ambient fluid (in general the ocean) of density $\rho_{w}$ generating a turbidity current. The models presented by the cited authors may be written under the following general formulation: 


$$
\left\{\begin{array}{l}
\partial_{t} h+\partial_{x}(h u)=\phi_{\eta}, \\
\partial_{t}(h u)+\partial_{x}\left(h u^{2}+g R_{c} \frac{h^{2}}{2}\right)=g\left(R_{0}+R_{c}\right) h \partial_{x} H+\tau, \\
\partial_{t}\left(h c_{j}\right)+\partial_{x}\left(h u c_{j}\right)=\phi_{b}^{j}, \text { for } j=1, \ldots, n_{s} \\
\partial_{t} H=\xi \phi_{b} .
\end{array}\right.
$$

where $h$ is the current thickness; $u$ is the depth-averaged velocity; $c_{j}$ for $j=1, \ldots, n_{s}$ represents the vertically averaged volume concentration of the $j$ th sediment; and $H$ is the bottom depth from a fixed level. Finally,

$$
R_{j}=\frac{\rho_{j}-\rho_{0}}{\rho_{0}}, \text { for } j=1, \ldots, n_{s} ; \quad \text { and } R_{c}=\sum_{j=1}^{n_{s}} R_{j} c_{j} .
$$

The source term $\phi_{\eta}$ represents the amount of ocean water entrained and mixed by turbulence to the plume.

The sediment flux at the bed of the $j$ th specie is determined from the rates of deposition $\left(F_{d}^{j}\right)$ and erosion $\left(F_{e}^{j}\right)$,

$$
\phi_{b}^{j}=F_{e}^{j}-F_{d}^{j}, \quad \phi_{b}=\sum_{j=1}^{n_{s}} \phi_{b}^{j} .
$$

We denote $\xi=1 /(1-\gamma)$, being $\gamma$ the porosity of the sediment layer.

Finally, $\tau$ represents the friction term.

While there are some discrepancies in the physical laws that describe the source terms $\phi_{\eta}$ and $\phi_{b}$, we shall use here the expressions that are the most extended. In particular, following [23], [17] and [14], the amount of ocean water entrained and mixed by turbulence to the plume, $\phi_{\eta}$, may be described as $E_{w} u$, where the water entrainment coefficient, $E_{w}$, is determined from the empirical relationship

$$
E_{w}=\frac{0.00153}{0.0204+R i},
$$

where the Richardson number, $R i$ is defined as

$$
R i=\frac{R_{c} g h}{u^{2}} .
$$

The rate of deposition is described as the product of the settling velocity of sediment, $v_{s_{j}}$, and the fractional concentration of suspension near by the bed, $c_{b_{j}}$. For multiple grain sizes, the sum of this product for each size population is used as the net rate of deposition

$$
F_{d}^{j}=v_{s_{j}} c_{b_{j}} .
$$

This definition is found for example in [23], [5] and [14] while other formulation is presented in [16]. The near bed concentration of sediment, $c_{b_{j}}$, can be related to the layer averaged concentration, $c_{j}$, by a factor 2, as it is the case in [12] and [2], or by the more extended expression used in [14] and [5].

$$
\frac{c_{b_{j}}}{c_{j}}=0.4\left(\frac{D_{j}}{D_{s g}}\right)^{1.64}+1.64
$$

where $D_{j}$ is the characteristic grain size and $D_{s g}$ denotes the geometric mean size of the suspended sediment mixture.

The rate of sediment entrainment from the bed is described as

$$
F_{e}^{j}=v_{s_{j}} p_{j} E_{s_{j}},
$$

where the sediment entrainment coefficient, $E_{s_{j}}$, is given by the expression developed by Garcia and Parker [11],

$$
E_{s_{j}}=\frac{1.3 \cdot 10^{-7} Z_{j}^{5}}{1+4.3 \cdot 10^{-7} Z_{j}^{5}}
$$


where

$$
Z_{j}=\alpha_{1} \frac{\sqrt{c_{D}}|u|}{v_{s_{j}}} \mathcal{R}_{p_{j}}^{\alpha_{2}}
$$

and

$$
\mathcal{R}_{p_{j}}=\frac{\sqrt{R_{j} g D_{j}} D_{j}}{\nu}
$$

is the particle Reynolds number, with $\nu$ the kinematic viscosity. The parameters $\left(\alpha_{1}, \alpha_{2}\right)$ take respective values $(1,0.6)$ for $\mathcal{R}_{p_{j}}>2.36$ and $(0.586,1.23)$ for $\mathcal{R}_{p_{j}} \leq 2.36$. We remark again that this is not the only expression used in models describing the physics of the problem. Some variants can be found in [5], [17] and [16].

In $(2.8), p_{j}$ represents the volume fraction of the $j$ th sediment in the bed. It could be described as a function of time as it is the case in $[5]$.

The friction term $\tau$ is described as a function of the velocity,

$$
\tau=-(1+\alpha) c_{D}|u| u
$$

$c_{D}$ is the bed drag coefficient which ranges from 0.002 to 0.05 depending on the flow type and $\alpha$ is the ratio of the drag force at the upper flow surface to that at the bed.

Finally, the fall velocity $v_{s_{j}}$ is calculated using the empirical relationship introduced by Dietrich [10]. We remark that there are some difficulties with system (2.1). First, let us denote by $c_{0}$ the freshwater concentration present in the plume and by $c$ the summation of all of the sediment fractions. The relation $c_{0}=1-c$ is satisfied and from $(2.1)$ one gets

$$
\partial_{t}\left(c_{0} h\right)+\partial_{x}\left(c_{0} h u\right)=\phi_{\eta}-\phi_{b}
$$

This means that erosion and deposition effects modify the freshwater mass while it is clear that in the case that there is no water entrainment from above, $\phi_{\eta}=0$, freshwater mass should be preserved. Another point is that deposition/erosion and water entrainment are not taken into account for the momentum equation. We will see in Section 3 that source terms related to deposition/erosion and water entrainment appear naturally in the momentum equation.

We would also like to take into account the ambient fluid considered and in particular its density $\rho_{w}$, so that we could model a plume that plunges into the ocean as well as a current flowing over a given bottom.

Finally, we would like to include into the model the sediment transport of bed-load particles due to the velocity of the current.

Taking into account these considerations, we are going to introduce the following model for hyperpycnal plumes:

$$
\left\{\begin{array}{l}
\partial_{t} h+\partial_{x}(h u)=\phi_{\eta}+\phi_{b}, \\
\partial_{t}(h u)+\partial_{x}\left(h u^{2}+g\left(R_{0}+R_{c}\right) \frac{h^{2}}{2}\right)=g\left(R_{0}+R_{c}\right) h \partial_{x} H+u \phi_{\eta}+\frac{u}{2} \phi_{b}+\tau, \\
\partial_{t}\left(h c_{j}\right)+\partial_{x}\left(h u c_{j}\right)=\phi_{b}^{j}, \text { for } j=1, \ldots, n_{s} \\
\partial_{t} H-\xi \partial_{x} q_{b}=\xi \phi_{b} .
\end{array}\right.
$$

Here,

$$
R_{0}=\frac{\rho_{0}-\rho_{w}}{\rho_{0}}
$$

Remark 2.1. In the general case we consider a river with a mixture of sediments that plunges into the ocean: $\rho_{w}$ (saltwater density) is slightly greater than $\rho_{0}$ (freshwater density) and $R_{0}<0$. The case where ambient fluid is air can also be considered: in that case $\rho_{w} \approx 0$ and $R_{0} \approx 1$.

Remark 2.2. The system (2.17) presents some major differences with respect to the model presented in [23], [5], and [14]. 
- From (2.17) one gets

$$
\partial_{t}\left(c_{0} h\right)+\partial_{x}\left(c_{0} h u\right)=\phi_{\eta},
$$

with $c_{0}=1-c$ the freshwater concentration. Thus, if there is no ocean water entrainment, the total freshwater mass is preserved.

- The pressure term $g R_{0} h^{2} / 2$ in the second equation allows to consider a turbid current that plunges into the ocean $\left(R_{0} \approx 0\right)$ as well as currents flowing over a given bottom $\left(R_{0} \approx 1\right)$.

- The effects of deposition/erosion and water entrainment on the momentum equation are taken into account.

- Solid transport of particles at the bottom is allowed via a solid transport flux $q_{b}$.

Taking into account the empirical relations described before, (2.14) reads

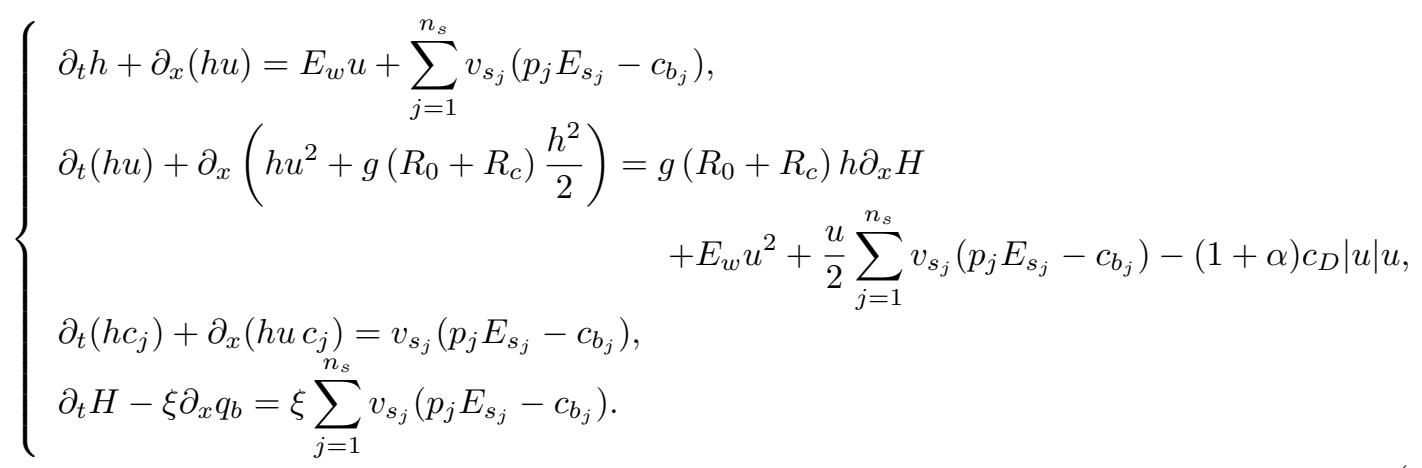

Sediment transport of bed-load particles due to the velocity of the current is represented by $\xi q_{b}$ where $\xi=1 /(1-\gamma)$, being $\gamma$ the porosity of the sediment layer and $q_{b}$ the solid transport flux, that depends on the fluid velocity $u, q_{b}=q_{b}(h, h u)$.

Several formulae for solid transport flux have been proposed, see [13], [18], [20], etc. Here, we shall consider the simplest one, which is the Grass model. According to it, the solid transport fluid equation is

$$
q_{b}=A_{g} u|u|^{m_{g}-1}, 1 \leq m_{g} \leq 4,
$$

where $A_{g}$ is a constant that represents the effects due to grain size and kinematic viscosity and is usually determined by experimental data. Usually, the constant $m_{g}$ is set to $m_{g}=3$.

One could also consider other expression for the solid transport flux and most of what is going to be said remains valid. The election of Grass model will only play an essential role in Lemma 4.3, where the explicit expresion of the corresponding eigenvalues can be given, as well as in the explicit definition of a Roe matrix in Section 5.

\section{Derivation of the layer-averaged equations}

\subsection{Governing equations for a dilute suspension}

We consider the situation described in Section 1 and, in particular, in Figure 1. We shall assume the rigid lid hypothesis for the ambient fluid in order to simulate just the turbidity current. The width of the plume is assumed to be small compared to the typical length phenomena in the horizontal direction so that the shallow water hypothesis can be made.

The equations of motion for a suspension are considered. The suspension is assumed to be sufficiently dilute to justify the use of the Boussinesq approximation, and the assumption of a kinematic viscosity $\nu$ equal to the value for clear water. The equation of momentum conservation is

$$
\frac{\partial \vec{u}}{\partial t}+\operatorname{div}(\vec{u} \otimes \vec{u})=-\frac{1}{\rho_{0}} \nabla p+\frac{1}{\rho_{0}} \nabla \cdot \mathcal{T}+\left(1+R_{c}\right) \vec{g}
$$


where $\vec{u}(\vec{x}, t)$ represents the instantaneous velocity of the mixture, $p(\vec{x}, t)$ denotes the instantaneous pressure and $\vec{g}=(0,0,-g)$ is the gravity and

$$
\mathcal{T}=\mu\left(\nabla \vec{u}+(\nabla \vec{u})^{t}\right)
$$

is the stress tensor. We denote $c=\sum_{j=1}^{n_{s}} c_{j}, R_{0}=1-\rho_{w} / \rho_{0}$, and $R_{c}=\sum_{j=1}^{n_{s}} R_{j} c_{j}$, where $R_{j}=$ $\rho_{j} / \rho_{0}-1$ and $c_{j}(x, t)$, for $j=1, \ldots, n_{s}$ denotes the instantaneous volumetric concentration of the $j$ th suspended sediment.

The fluid is assumed to be incompressible and the continuity equation is also considered:

$$
\begin{aligned}
& \operatorname{div} \vec{u}=0, \\
& \partial_{t} \rho+\operatorname{div}(\rho \vec{u})=0,
\end{aligned}
$$

where $\rho$ is the density of the turbidity current.

Following the notation of Section 2, we may rewrite the density of the current as

$$
\rho=\sum_{j=0}^{n_{s}}\left(\rho_{j} c_{j}\right)=\rho_{0}\left(1+\sum_{j=1}^{n_{s}} R_{j} c_{j}\right)
$$

where $c_{0}(\vec{x}, t)$ denotes the portion of water in the turbidity current.

Now, as $\sum_{j=0}^{n_{s}} c_{j}=1$, from equations (3.3) and (3.4) we get

$$
0=\partial_{t}\left(c_{0} \rho_{0}+\sum_{j=1}^{n_{s}} \rho_{j} c_{j}\right)+\operatorname{div}\left(\left(c_{0} \rho_{0}+\sum_{j=1}^{n_{s}} c_{j} \rho_{j}\right) \vec{u}\right)=\partial_{t}\left(\sum_{j=1}^{n_{s}} c_{j}\left(\rho_{j}-\rho_{0}\right)\right)+\operatorname{div}\left(\sum_{j=1}^{n_{s}} c_{j}\left(\rho_{j}-\rho_{0}\right) \vec{u}\right) .
$$

In order to satisfy (3.6), we shall assume that the volume fraction of each sediment specie satisfies the equation

$$
\frac{\partial c_{j}}{\partial t}+\operatorname{div}\left(c_{j} \vec{u}\right)=0, \quad \text { for } j=1, \ldots, n_{s} .
$$

As a consequence, this relation is also satisfied by the volume fraction of fresh water and the equations of mass conservation are thus

$$
\frac{\partial c_{j}}{\partial t}+\operatorname{div}\left(c_{j} \vec{u}\right)=0, \quad \text { for } j=0,1, \ldots, n_{s} .
$$

\subsection{Vertically integrated equations}

Henceforth $\vec{x}=(x, y, z)$ and $\vec{u}=(u, v, w)$. Variations in the horizontal $y$ direction are neglected and we suppose $v=0$. It is assumed that the thickness of the turbidity current is small compared to the typical length phenomena in the horizontal direction so that the shallow water hypothesis can be made.

As the current is essentially two-dimensional, the domain considered is

$$
\left\{(t, x, z) \in \mathbb{R}^{+} \times \mathbb{R}^{2} / b(x, t)<z<b(x, t)+h(x, t)\right\},
$$

where $b(x, t)$ is the bottom elevation from a defined reference level and $h(x, t)$ is the length of the turbidity layer.

As it has been said, perturbations of the free surface due to the turbidity current are neglected, so that the water surface is considered to be constant and equal to $z=H_{0}$.

It will be useful to define the bottom depth from the surface

$$
H(x, t)=H_{0}-b(x, t),
$$


and the interface between the turbidity current and the ambient water

$$
\eta(x, t)=b(x, t)+h(x, t) .
$$

Some kinematic conditions should be imposed at the bottom and at the interface. The turbidity current lies between an upper non-material interface, $F^{s}=z-\eta(x, t)=0$, and a basal interface, $F^{b}=b(x, t)-z=0$. These curves are defined so that their unit normals $n^{s}=\nabla F^{s} /\left|\nabla F^{s}\right|$ and $n^{b}=\nabla F^{b} /\left|\nabla F^{b}\right|$ point outwards from the turbidity current. Assuming that these interfaces have velocities $\vec{v}^{s}$ and $\vec{v}^{b}$, the kinematic boundary conditions are

$$
\begin{array}{ll}
F^{s}(x, z, t)=0: & \partial_{t} F^{s}+\vec{v}^{s} \cdot \nabla F^{s}=0, \\
F^{b}(x, z, t)=0: & \partial_{t} F^{b}+\vec{v}^{b} \cdot \nabla F^{b}=0 .
\end{array}
$$

The upper and basal interfaces are modified by entrainment of water from the above and deposition/erosion of sediment at the bottom. Let the normal entrainment rate, $d^{s}$, be the equivalent volume of ambient water entrained to the turbid current at the upper interface per unit area per unit time. Then the velocity of the interface is $\vec{v}^{s}=\vec{u}^{s}+d^{s} \vec{n}^{s}$. Similarly, if the normal deposition/erosion rate, $d^{b}$, is the equivalent volume of granular material deposited/eroded at the bottom per unit area per unit time, the velocity of the basal interface is $\vec{v}^{b}=\vec{u}^{b}+d^{b} \vec{n}^{b}$. It follows that the kinematic conditions (3.12)-(3.13) are

$$
\begin{array}{ll}
z=\eta(x, t): & \partial_{t} \eta+u^{s} \partial_{x} \eta-w^{s}=\left(1+\left(\partial_{x} \eta\right)^{2}\right)^{1 / 2} d^{s}, \\
z=b(x, t): & \partial_{t} b+u^{b} \partial_{x} b-w^{b}=-\left(1+\left(\partial_{x} b\right)^{2}\right)^{1 / 2} d^{b} .
\end{array}
$$

Thus, we shall consider the kinematic equations

$$
\begin{gathered}
\partial_{t} \eta+\left.u\right|_{z=\eta} \partial_{x} \eta-\left.w\right|_{z=\eta}=\widetilde{\phi}_{\eta}, \\
\partial_{t} b+\left.u\right|_{z=b} \partial_{x} b-\left.w\right|_{z=b}=-\widetilde{\phi}_{b},
\end{gathered}
$$

where $\vec{u}=(u, w), \widetilde{\phi}_{\eta}=\left(1+\left(\partial_{x} \eta\right)^{2}\right)^{1 / 2} d^{s}$, and $\widetilde{\phi}_{b}=\left(1+\left(\partial_{x} b\right)^{2}\right)^{1 / 2} d^{b}$.

Under these hypothesis, mass conservation equations yield

$$
\partial_{t} \int_{b}^{\eta} c_{j} d z+\partial_{x} \int_{b}^{\eta} c_{j} u d z=\left.c_{j}\right|_{z=\eta} \widetilde{\phi}_{\eta}+\left.c_{j}\right|_{z=b} \widetilde{\phi}_{b}, \text { for } j=0,1, \ldots, n_{s} .
$$

We shall only retain the vertical components of the stress tensor and assume that the other terms may be neglected. Let us denote by

$$
\widetilde{\tau}=\frac{\mu}{\rho_{0}}\left(\frac{\partial u}{\partial z}+\frac{\partial w}{\partial x}\right)
$$

the $(1,2)$ component of the stress tensor $\mathcal{T}$. Let us also suppose that the pressure $p$ reduces to hydrostatic effects,

$$
p(x, z, t)=\int_{z}^{\eta} \sum_{j=0}^{n_{s}}\left(\rho_{j} c_{j}\right) g d z^{\prime}+g \rho_{w}\left(H_{0}-h-b\right) .
$$

By integrating the momentum equation, we obtain:

$$
\partial_{t} \int_{b}^{\eta} u d z+\partial_{x} \int_{b}^{\eta} u^{2} d z+\frac{g}{\rho_{0}} \int_{b}^{\eta} \partial_{x} p d z-g \frac{\rho_{w}}{\rho_{0}} h \partial_{x} \eta=\left.\widetilde{\tau}\right|_{z=\eta}-\left.\widetilde{\tau}\right|_{z=b}+\left.u\right|_{z=\eta} \widetilde{\phi}_{\eta}+\left.u\right|_{z=b} \widetilde{\phi}_{b} .
$$

Definition 3.1. For any function $f(x, z, t)$ we define the mean of the function as

$$
\bar{f}=\frac{1}{h} \int_{b}^{\eta} f d z
$$

The fluctuating part of $f$ relative to the mean is defined by

$$
\widehat{f}=f-\bar{f} .
$$


Using this notation, it is clear now that for any functions $f_{1}, f_{2}$ one has

$$
h \overline{f_{1}} \overline{f_{2}}+\int_{b}^{\eta} \widehat{f_{1}} \widehat{f_{2}} d z=\int_{b}^{\eta} f_{1} f_{2} d z .
$$

Thus, equation (3.18) writes as follows:

$$
\partial_{t}\left(h \overline{c_{j}}\right)+\partial_{x}\left(h \overline{c_{j}} \bar{u}\right)+\partial_{x}\left(\int_{b}^{\eta} \widehat{c_{j}} \widehat{u_{j}} d z\right)=\left.c_{j}\right|_{z=\eta} \widetilde{\phi}_{\eta}+\left.c_{j}\right|_{z=b} \widetilde{\phi}_{b} \text {, for } j=0,1, \ldots, n_{s} .
$$

From equation (3.20) we get

$$
p(x, z, t)=g \sum_{j=0}^{n_{s}}\left(\rho_{j} \overline{c_{j}}(\eta-z)\right)+g \rho_{w}\left(H_{0}-h-b\right)+g \int_{z}^{\eta} \sum_{j=0}^{n_{s}}\left(\rho_{j} \widehat{c_{j}}\right) d z^{\prime},
$$

and

$$
\begin{aligned}
\int_{b}^{\eta} \frac{1}{\rho_{0}} \partial_{x} p d z & -g \int_{b}^{\eta} \partial_{x}\left\{\int_{z}^{\eta} \sum_{j=0}^{n_{s}}\left(\rho_{j} \widehat{c_{j}}\right) d z^{\prime}\right\} d z \\
& =\frac{g}{\rho_{0}} \int_{b}^{\eta} \partial_{x}\left(\sum_{j=0}^{n_{s}}\left(\rho_{j} \overline{c_{j}}\right)(\eta-z)\right) d z-g \frac{\rho_{w}}{\rho_{0}} h \partial_{x}(h+b) \\
& =\frac{g}{\rho_{0}} \partial_{x}\left(\int_{b}^{\eta} \sum_{j=0}^{n_{s}}\left(\rho_{j} \overline{c_{j}}(\eta-z)\right) d z\right)+\frac{g}{\rho_{0}} \sum_{j=0}^{n_{s}} \rho_{j} \overline{c_{j}} h \partial_{x} b-g \frac{\rho_{w}}{\rho_{0}} h \partial_{x}(h+b) \\
& =g \partial_{x}\left(R_{0}+\sum_{j=1}^{n_{s}} R_{j} \overline{c_{j}} \frac{h^{2}}{2}\right)+g\left(R_{0}+\sum_{j=1}^{n_{s}} R_{j} \overline{c_{j}}\right) h \partial_{x} b .
\end{aligned}
$$

(3.21) can be thus written as follows:

$$
\begin{aligned}
\partial_{t}(h \bar{u}) & +\partial_{x}\left(h \bar{u}^{2}+g\left(R_{0}+\sum_{j=1}^{n_{s}} R_{j} \overline{c_{j}}\right) \frac{h^{2}}{2}\right)+g\left(R_{0}+\sum_{j=1}^{n_{s}} R_{j} \overline{c_{j}}\right) h \partial_{x} b \\
& +\int_{b}^{\eta} \widehat{u}^{2} d z+g \int_{b}^{\eta} \partial_{x} \int_{z}^{\eta} \sum_{j=0}^{n_{s}}\left(\rho_{j} \widehat{c_{j}}\right) d z^{\prime} d z=\left.\widetilde{\tau}\right|_{z=\eta}-\left.\widetilde{\tau}\right|_{z=b}+\left.u\right|_{z=\eta} \widetilde{\phi}_{\eta}+\left.u\right|_{z=b} \widetilde{\phi}_{b} .
\end{aligned}
$$

\subsection{Similarity assumptions}

In this point, it is necessary to make some assumptions in order to write the integrated equations in terms of mean variables. We will suppose that the variables $c_{j}$ maintain approximately similar profiles in the z-direction so that the terms $\widehat{c_{j}}$ may be neglected. The term $\int_{b}^{\eta} \widehat{u}^{2} d z$ corresponds to the

kinematic Reynolds stress. We shall assume that this term and the tensor stress may be expressed in terms of $\bar{u}$ so that we write

$$
\left.\widetilde{\tau}\right|_{z=\eta}-\left.\widetilde{\tau}\right|_{z=b}-\int_{b}^{\eta} \widehat{u}^{2} d z=\tau(\bar{u}) .
$$

Let us consider the approximations

$$
\left.\phi_{\eta}^{j} \simeq c_{j}\right|_{z=\eta} \widetilde{\phi}_{\eta},\left.\quad \phi_{b}^{j} \simeq c_{j}\right|_{z=b} \widetilde{\phi}_{b}, \quad j=0,1, \ldots, n_{s},
$$

and

$$
\phi_{\eta}=\sum_{j=0}^{n_{s}} \phi_{\eta}^{j} \simeq \widetilde{\phi}_{\eta}, \quad \phi_{b}=\sum_{j=0}^{n_{s}} \phi_{b}^{j} \simeq \widetilde{\phi}_{b} .
$$


In particular, as there is no water entrainment at the bottom, we shall assume $\phi_{b}^{0}=0$, and $\phi_{b}^{j}$ for $j=1, \ldots, n_{s}$ will be given by the empirical relations described in Section 2 . In the same way, there is no sediment transfer at the interface $z=\eta$ and we shall suppose $\phi_{\eta}^{j}=0$, for $j=1, \ldots, n_{s}$ and $\phi_{\eta}^{0} \equiv \phi_{\eta}$ described in Section 2 .

Following [4], we shall assume $\left.u\right|_{z=\eta}=\bar{u}$ and $\left.u\right|_{z=b}=\frac{1}{2} \bar{u}$.

Thus, using the fact that $c_{0}=1-c$ and $\partial_{x} b=-\partial_{x} H$ we arrive to the final model

$$
\left\{\begin{array}{l}
\partial_{t} h+\partial_{x}(h \bar{u})=\phi_{\eta}+\phi_{b}, \\
\partial_{t}(h \bar{c})+\partial_{x}(h \bar{u} \bar{c})=\phi_{b}, \\
\partial_{t}(h \bar{u})+\partial_{x}\left(h \bar{u}^{2}+g\left(R_{0}+R_{c}\right) \frac{h^{2}}{2}\right)=g\left(R_{0}+R_{c}\right) h \partial_{x} H+\bar{u} \phi_{\eta}+\frac{\bar{u}}{2} \phi_{b}+\tau .
\end{array}\right.
$$

The second equation will be replaced by one equation for each sediment specie,

$$
\partial_{t}\left(h \overline{c_{j}}\right)+\partial_{x}\left(h \bar{u} \overline{c_{j}}\right)=\phi_{b}^{j}, \text { for } j=1, \ldots, n_{s},
$$

where $\sum_{j=1}^{n_{s}} \phi_{b}^{j}=\phi_{b}$.

\subsection{Evolution of the topography}

Sediment and erosion of the bottom as well as sediment transport due to the velocity of the current are considered here. The bed-sediment conservation equation has the form

$$
\partial_{t} b+\xi \partial_{x} q_{b}=-\xi \phi_{b}
$$

where $q_{b}=q_{b}(h, h \bar{u})$ denotes the solid transport flow and $\xi=\frac{1}{1-\gamma}$, where $\gamma$ is the porosity of the bed.

\section{Reformulation and properties of the model}

The system may be rewritten in the form:

$$
\partial_{t} W+\partial_{x} \tilde{F}(W)=B(W) \partial_{x} W+S(W),
$$

where $W=\left(h, h u, h c_{1}, \ldots, h c_{n_{s}}, H\right)^{t}, \widetilde{F}(W)=\left(h u, h u^{2}+g / 2\left(R_{0}+R_{c}\right) h^{2}, h c_{1} u, \ldots, h c_{n_{s}} u,-\xi q_{b}\right)^{t}$, $B(W)=g\left(R_{0}+R_{c}\right) h e_{2, n_{s}+3}$ and

$$
S(W)=\left(\begin{array}{c}
E_{w} u+\sum_{j=1}^{n_{s}} v_{s_{j}}\left(p_{j} E_{s_{j}}-c_{b_{j}}\right) \\
E_{w} u^{2}+\frac{u}{2} \sum_{j=1}^{n_{s}} v_{s_{j}}\left(p_{j} E_{s_{j}}-c_{b_{j}}\right)-(1+\alpha) c_{D}|u| u \\
v_{s_{1}}\left(p_{1} E_{s_{1}}-c_{b_{1}}\right) \\
\ldots \\
v_{s_{n_{s}}}\left(p_{n_{s}} E_{s_{n_{s}}}-c_{b_{n_{s}}}\right) \\
\xi \sum_{j=1}^{n_{s}} v_{s_{j}}\left(p_{j} E_{s_{j}}-c_{b_{j}}\right)
\end{array}\right)
$$

Here $e_{r, s}=\left(\delta_{i r} \delta_{j s}\right)_{i=1, j=1}^{n_{s}+3, n_{s}+3}$ is the canonical basis of square matrices of order $n_{s}+3$.

We will also use the notation $U=\left(h, h u, h c_{1}, \ldots, h c_{n_{s}}\right)^{t}, F(U)=\left(h u, h u^{2}+g / 2\left(R_{0}+R_{c}\right) h^{2}, h c_{1} u, \ldots, h c_{n_{s}} u\right)^{t}$. The problem may also be written in the more general form

$$
\partial_{t} W+\mathcal{A}(W) \partial_{x} W=S(W)
$$

where $\mathcal{A}=\nabla \widetilde{F}(W)-B(W)$. More explicitly,

$$
\mathcal{A}(W)=\left(\begin{array}{ccccccc}
0 & 1 & 0 & 0 & \ldots & 0 & 0 \\
g h\left(R_{0}+\frac{1}{2} R_{c}\right)-u^{2} & 2 u & \frac{g}{2} R_{1} h & \frac{g}{2} R_{2} h & \ldots & \frac{g}{2} R_{n_{s}} h & -g\left(R_{0}+R_{c}\right) h \\
-c_{1} u & c_{1} & u & 0 & \ldots & 0 & 0 \\
-c_{2} u & c_{2} & 0 & u & \ldots & 0 & 0 \\
\ldots & \ldots & \ldots & \ldots & \ldots & \ldots & \ldots \\
-c_{n_{s}} u & c_{n_{s}} & 0 & 0 & \ldots & u & \ldots \\
-\xi \frac{\partial q_{b}}{\partial h} & -\xi \frac{\partial q_{b}}{\partial(h u)} & 0 & 0 & \ldots & 0 & 0
\end{array}\right)
$$


Theorem 4.1. Suppose $R_{0} h+R_{c} h>0$ and $\nabla q_{b}$ sufficiently small. Then, the system (4.3) is hyperbolic. Moreover, even though it is not strictly hyperbolic for $n_{s}>1$, one can always find a complete set of eigenvector for $\mathcal{A}$ in $\mathbb{R}^{n_{s}+3}$.

Proof.

This is a consequence of the following lemma.

Lemma 4.2. Consider the matrix

$$
\mathcal{A}=\left(\begin{array}{ccccccc}
0 & 1 & 0 & 0 & \ldots & 0 & 0 \\
g\left(R_{0} h+\frac{1}{2} \gamma\right)-u^{2} & 2 u & \frac{g}{2} R_{1} h & \frac{g}{2} R_{2} h & \ldots & \frac{g}{2} R_{n_{s}} h & -g\left(R_{0} h+\gamma\right) \\
-c_{1} u & c_{1} & u & 0 & \ldots & 0 & 0 \\
-c_{2} u & c_{2} & 0 & u & \ldots & 0 & 0 \\
\ldots & \ldots & \ldots & \ldots & \ldots & \ldots & \ldots \\
-c_{n_{s}} & c_{n_{s}} & 0 & 0 & \ldots & u & 0 \\
\alpha & \beta & 0 & 0 & \ldots & 0 & 0
\end{array}\right)
$$

and suppose $R_{0} h+\frac{\gamma}{2}+\frac{1}{2} R_{c} h>0$. There exists $\varepsilon>0$ such that if $\alpha^{2}+\beta^{2}<\varepsilon$, then the the matrix $\mathcal{A}$ has $n_{s}+3$ real eigenvalues, being $\lambda=u$ an eigenvalue of multiplicity at least $n_{s}$. Moreover, if there are four different eigenvalues, a basis of eigenvectors can be found.

\section{Proof.}

Some easy calculations show that the characteristic polynomial of $\mathcal{A}$ is

$$
p(\lambda)=-(u-\lambda)^{n_{s}} q(\lambda),
$$

where

$$
\begin{gathered}
q(\lambda)=\lambda^{3}+a_{2} \lambda^{2}+a_{1} \lambda+a_{0}, \\
a_{2}=-2 u, \\
a_{1}=u^{2}-g\left(R_{0} h+\frac{\gamma}{2}+\frac{1}{2} R_{c} h\right)+g\left(R_{0} h+\gamma\right) \beta, \\
a_{0}=g\left(R_{0} h+\gamma\right) \alpha .
\end{gathered}
$$

Thus, $u$ is an eigenvalue of multiplicity at least $n_{s}$.

In the particular case $\alpha=\beta=0$, the eigenvalues are

$$
\begin{gathered}
\lambda_{1}=u-\sqrt{g\left(R_{0} h+\frac{\gamma}{2}+\frac{1}{2} R_{c} h\right)}, \\
\lambda_{2}=u+\sqrt{g\left(R_{0} h+\frac{\gamma}{2}+\frac{1}{2} R_{c} h\right)}, \\
\lambda_{2+j}=u, j=1, \ldots, n_{s}, \\
\lambda_{n_{s}+3}=0 .
\end{gathered}
$$

By continuity, the matrix has $n_{s}+3$ real eigenvalues for $\alpha^{2}+\beta^{2}$ sufficiently small.

Now, denote by $\lambda_{1}, \lambda_{2}, \lambda_{3}$ the roots of the polynomial $q(\lambda)$ and assume they are different real numbers not equal to $u$.

If $r=\left(x, y, w_{1}, \ldots, w_{n}, z\right)^{t}$ is an eigenvector of $\mathcal{A}$ corresponding to the eigenvalue $\lambda$, then

$$
\left\{\begin{array}{l}
y=\lambda x \\
\left(\lambda^{2}+u^{2}-g\left(R_{0} h+\frac{\gamma}{2}\right)-2 u \lambda\right) x=\sum_{j=1}^{n_{s}} \frac{g}{2} R_{j} w_{j} h-g\left(R_{0} h+\gamma\right) z, \\
c_{j}(\lambda-u) x=(\lambda-u) w_{j}, \text { for } j=1, \ldots, n_{s}, \\
(\alpha+\beta \lambda) x=\lambda z
\end{array}\right.
$$

We shall consider some different cases: 
A) Case $\lambda \in\left\{\lambda_{1}, \lambda_{2}, \lambda_{3}\right\}$

A.1) Case $\lambda \neq 0$

As $\lambda$ is a root of the polynomial $q$, one has

$$
(\lambda-u)^{2}-g\left(R_{0} h+\frac{\gamma}{2}+\frac{1}{2} R_{c} h\right)=-g\left(R_{0} h+\gamma\right)(\alpha+\beta \lambda) \lambda^{-1} .
$$

Thus, the system (4.15) reduces to

$$
\left\{\begin{array}{l}
y=\lambda x \\
\left(R_{0} h+\gamma\right)(\alpha+\beta \lambda) \lambda^{-1} x=\left(R_{0} h+\gamma\right) z \\
c_{j} x=w_{j}, \text { for } j=1, \ldots, n_{s} \\
(\alpha+\beta \lambda) x=\lambda z
\end{array}\right.
$$

and one can easily check that

$$
r=\left(\lambda, \lambda^{2}, \lambda c_{1}, \ldots, \lambda c_{n}, \alpha+\beta \lambda\right)^{t} .
$$

is an eigenvector.

A.2) Case $\lambda=0$

In this case, we have $a_{0}=g\left(R_{0} h+\gamma\right) \alpha=0$ and system (4.15) reduces to

$$
\left\{\begin{array}{l}
y=0 \\
\left(u^{2}-g\left(R_{0} h+\frac{\gamma}{2}+\frac{1}{2} R_{c} h\right)\right) x=-g\left(R_{0} h+\gamma\right) z \\
c_{j} x=w_{j}, \text { for } j=1, \ldots, n_{s} \\
\alpha x=0
\end{array}\right.
$$

We set

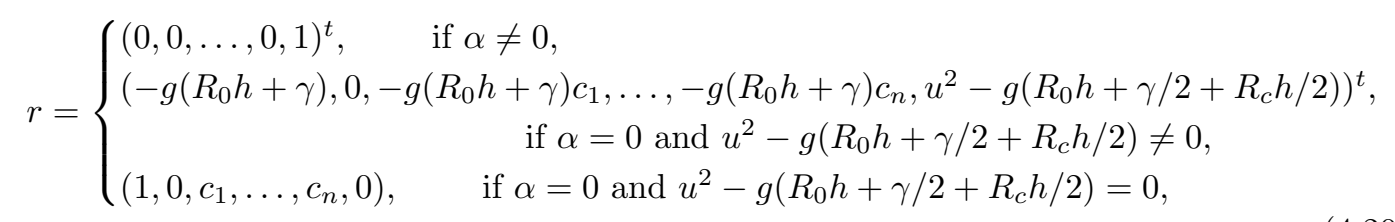

which defines an eigenvector for $\lambda=0$.

B) Case $\lambda=u$

Any eigenvector associated to $u$ should verify

$$
\left\{\begin{array}{l}
y=u x \\
-\left(R_{0} h+\frac{\gamma}{2}\right) x=\frac{1}{2} \sum_{j=1}^{n_{s}} R_{j} w_{j} h-\left(R_{0} h+\gamma\right) z \\
(\alpha+\beta u) x=u z
\end{array}\right.
$$

Thus, define for $j=1, \ldots, n_{s}$

$$
r_{j}=\frac{1}{2} R_{j} h\left(u, u^{2}, 0, \ldots, 0, \alpha+\beta u\right)+\left((\alpha+\beta u)\left(R_{0} h+\gamma\right)-u\left(R_{0} h+\frac{\gamma}{2}\right)\right) e_{j+2},
$$

where $e_{i}, i=1, \ldots, n_{s}+3$ represents the $i$ th element of the canonical basis in $\mathbb{R}^{n_{s}+3}$. They form a set of $n_{s}$ independent eigenvectors corresponding to $u$ as long as $(\alpha+\beta u)\left(R_{0} h+\gamma\right)-u\left(R_{0} h+\frac{\gamma}{2}\right) \neq 0$. Now, if $(\alpha+\beta u)\left(R_{0} h+\gamma\right)=u\left(R_{0} h+\frac{\gamma}{2}\right)$, then $u \neq 0$ (otherwise, we would have $a_{0}=0$ and $u=0$ of multiplicity at least $n_{s}+1$ so there are only 3 different eigenvalues). Thus, system (4.15) writes

$$
\left\{\begin{array}{l}
y=u x \\
\sum_{j=1}^{n_{s}} R_{j} w_{j} h=0 \\
(\alpha+\beta u) x=u z
\end{array}\right.
$$


and we may define a set of $n_{s}$ independent eigenvectors by

$$
r_{1}=\left(u, u^{2}, 0, \ldots, 0, \alpha+\beta u\right),
$$

and

$$
r_{j}=r_{1}-R_{j} e_{j+1}+R_{j-1} e_{j+2}, \text { for } j=2, \ldots, n_{s} .
$$

Lemma 4.3. Suppose that the solid transport flux $q_{b}$ is given by the Grass model (2.18). Then if $R_{0}+R_{c}>0$ all the eigenvalues of (4.4) are real.

\section{Proof.}

In this particular case, the coefficients of the polynomial (4.7) are

$$
\begin{gathered}
a_{2}=-2 u, \\
a_{1}=u^{2}-g_{c} h(1+d), \\
a_{0}=g_{c} h u d,
\end{gathered}
$$

where $g_{c}=g\left(R_{0}+R_{c}\right)$ and $d=\xi \frac{\partial q_{b}}{\partial(h u)}=\xi \frac{1}{h} A_{g} m_{g}|u|^{m_{g}-1}$.

According to the Cardano-Vieta relations, the roots of $q(\lambda)$ are real if $Q^{3}+R^{2}<0$, where

$$
Q=\frac{3 a_{1}-a_{2}^{2}}{9}, \quad R=\frac{9 a_{1} a_{2}-27 a_{0}-2 a_{2}^{3}}{54} .
$$

In that case, the eigenvalues are given by

$$
\lambda_{i}=2 \sqrt{-Q} \cos \left(\frac{\theta+2^{i-1} \pi}{3}\right)-\frac{a_{2}}{3}, \quad i=1,2,3,
$$

with

$$
\theta=\arccos \left(\frac{R}{\sqrt{-Q^{3}}}\right)
$$

Some simple calculations show that

$$
\begin{gathered}
Q=\frac{-1}{9}\left(u^{2}+3 g_{c} h(d+1)\right), \\
R=\frac{u}{54}\left(9 g_{c} h(2-d)-2 u^{2}\right), \\
Q^{3}=-\frac{1}{3^{6}}\left(u^{6}+3^{2} u^{4} g_{c} h(d+1)+3^{3} u^{2} g_{c}^{2} h^{2}(d+1)^{2}+3^{3} g_{c}^{3} h^{3}(d+1)^{3},\right. \\
R^{2}=\frac{u^{2}}{2^{2} \cdot 3^{6}}\left(3^{4} g_{c}^{2} h^{2}(2-d)^{2}+2^{2} u^{4}-2^{2} \cdot 3^{2} u^{2} g_{c} h(2-d)\right) .
\end{gathered}
$$

Thus, $Q^{3}+R^{2}<0$ as long as

$$
\begin{aligned}
3^{4} g_{c}^{2} h^{2} u^{2}(2-d)^{2}-3^{2} \cdot & 2^{2} u^{4} g_{c} h(2-d) \\
& <3^{2} \cdot 2^{2} u^{4} g_{c} h(d+1)+3^{3} \cdot 2^{2} u^{2} h^{2} g_{c}^{2}(d+1)^{2}+3^{3} \cdot 2^{2} g_{c}^{3} h^{3}(d+1)^{3},
\end{aligned}
$$

which is equivalent to

$$
8 g_{c}^{2} h^{2} u^{2}<2^{2} g_{c} h u^{4}+g_{c}^{2} h^{2} u^{2} d(14+d)+2^{2} g_{c}^{3} h^{3}(d+1)^{3} .
$$


By hypothesis $g_{c}>0$. Thus, (4.37) is equivalent to

$$
\begin{aligned}
0<-8 g_{c} h^{2} u^{2}+2^{2} h u^{4}+g_{c} h^{2} u^{2} d(14+d)+2^{2} g_{c}^{2} h^{3}(d+1)^{3} & \\
& =4 h\left(u^{2}-g_{c} h\right)^{2}+g_{c} h d(14+d)+4 g_{c}^{2} h^{3}\left(d^{3}+3 d^{2}+3 d\right)
\end{aligned}
$$

and the result follows.

Proposition 4.4. Consider the system

$$
\begin{aligned}
& \partial_{t} h+\partial_{x}(h u)=\phi_{\eta}+\phi_{b}, \\
& \partial_{t}(h u)+\partial_{x}\left(h u^{2}+g\left(R_{0}+R_{c}\right) \frac{h^{2}}{2}\right)=g\left(R_{0}+R_{c}\right) h \partial_{x} H+u \phi_{\eta}+\frac{u}{2} \phi_{b}+\tau, \\
& \partial_{t}\left(h c_{j}\right)+\partial_{x}\left(h u c_{j}\right)=\phi_{b}^{j}, \text { for } j=1, \ldots, n_{s}, \\
& \partial_{t} H=\phi_{b} .
\end{aligned}
$$

Any smooth solution of (4.39) satisfies the equation

$$
\begin{aligned}
& \partial_{t}(\eta(W))+\partial_{x}(G(W))= \\
& \left(\frac{u^{2}}{2}+g\left(R_{0}(h-H)+\frac{R_{c} h}{2}\right)\right) \phi_{\eta}-g \frac{R_{c} h}{2} \phi_{b}+g\left(\frac{h}{2}-H\right) \sum_{j=1}^{n_{s}} R_{j} \phi_{b}^{j}+u \tau,
\end{aligned}
$$

with

$$
\eta(W)=h \frac{u^{2}}{2}+g\left(R_{0}+R_{c}\right) \frac{h^{2}}{2}-g\left(R_{0}+R_{c}\right) h H+g R_{0} \frac{H^{2}}{2}
$$

and

$$
G(W)=h u\left(\frac{u^{2}}{2}+g\left(R_{0}+g R_{c}\right)(h-H)\right) .
$$

\section{Proof.}

First, from equations (4.39a) and (4.39b) we get:

$$
\partial_{t} u+\partial_{x}\left(\frac{u^{2}}{2}\right)+\frac{1}{h} \partial_{x}\left(g\left(R_{0}+R_{c}\right) \frac{h^{2}}{2}\right)=g\left(R_{0}+R_{c}\right) \partial_{x} H-\frac{1}{2} \frac{u}{h} \phi_{b}+\frac{\tau}{h} .
$$

Now, multiplying equation (4.39a) by $\frac{u^{2}}{2}+g R_{0}(h-H)$ and equation (4.43) by $h u$ and adding the results we get

$$
\begin{aligned}
\partial_{t}\left(h \frac{u^{2}}{2}+g R_{0}\left(\frac{h^{2}}{2}-H h\right)\right)+\partial_{x}\left(h u\left(\frac{u^{2}}{2}+g R_{0}(h-H)\right)\right)+u \partial_{x}\left(g R_{c} \frac{h^{2}}{2}\right) \\
=g h u R_{c} \partial_{x} H-\frac{u^{2}}{2} \phi_{b}+\left(\frac{u^{2}}{2}+g R_{0}(h-H)\right)\left(\phi_{\eta}+\phi_{b}\right)-g R_{0} h \partial_{t} H+u \tau .
\end{aligned}
$$

The third term of the right hand side can be rewritten as follows: 


$$
\begin{aligned}
u \partial_{x}\left(g R_{c} \frac{h^{2}}{2}\right)=\partial_{x}\left(g R_{c} \frac{h^{2}}{2} u\right)-g R_{c} \frac{h}{2} \partial_{x}(h u)+g R_{c} \frac{h}{2} u \partial_{x} h \\
=\partial_{x}\left(g R_{c} \frac{h^{2}}{2} u\right)+g R_{c} \frac{h}{2}\left(\partial_{t} h-\left(\phi_{\eta}+\phi_{b}\right)\right)+g R_{c} \frac{h}{2} u \partial_{x} h+g \frac{h}{2} \sum_{j=1}^{n_{s}} R_{j}\left(\partial_{t}\left(c_{j} h\right)+\partial_{x}\left(h c_{j} u\right)-\phi_{b}^{j}\right) \\
\quad \partial_{t}\left(g R_{c} \frac{h^{2}}{2}\right)+\partial_{x}\left(g R_{c} u h^{2}\right)-\frac{g}{2} R_{c} h\left(\phi_{\eta}+\phi_{b}\right)-\frac{g}{2} h \sum_{j=1}^{n_{s}} R_{j} \phi_{b}^{j} .
\end{aligned}
$$

Using this expression in (4.44) we get

$$
\begin{aligned}
\partial_{t}\left(h \frac{u^{2}}{2}+g\left(R_{0}+R_{c}\right) \frac{h^{2}}{2}-g R_{0} H h\right)+ & \partial_{x}\left(h u\left(\frac{u^{2}}{2}+g\left(R_{0}+R_{c}\right) h-g R_{0} H\right)\right) \\
=g R_{c} h u \partial_{x} H & -g R_{0} h \partial_{t} H+g R_{0}(h-H)\left(\phi_{\eta}+\phi_{b}\right) \\
& +\left(\frac{u^{2}}{2}+g R_{c} \frac{h}{2}\right) \phi_{\eta}+\frac{g}{2} R_{c} h \phi_{b}+\frac{g}{2} h \sum_{j=1}^{n_{s}} R_{j} \phi_{b}^{j}+u \tau .
\end{aligned}
$$

Now, we remark

$$
\begin{aligned}
& \quad g R_{c} h u \partial_{x} H-g R_{0} h \partial_{t} H+g R_{0}(h-H)\left(\phi_{\eta}+\phi_{b}\right) \\
& =g R_{c} h u \partial_{x} H+g R_{0} h\left(\phi_{b}-\partial_{t} H\right)-g R_{0} H\left(\phi_{\eta}+\phi_{b}\right)+g R_{0} h \phi_{\eta}+g H \sum_{j=1}^{n_{s}} R_{j}\left(\partial_{t}\left(h c_{j}\right)+\partial_{x}\left(h c_{j} u\right)-\phi_{b}^{j}\right) \\
& \quad=\partial_{t}\left(g R_{c} h H\right)+\partial_{x}\left(g R_{c} h u H\right)-g R_{c} h \partial_{t} H-g R_{0} H\left(\phi_{\eta}+\phi_{b}\right)+g R_{0} h \phi_{\eta}-g H \sum_{j=1}^{n_{s}} R_{j} \phi_{b}^{j} . \quad \text { (4.47) }
\end{aligned}
$$

Using this result in (4.46) we obtain (4.40).

Corollary 4.5. Consider the system (4.39) and suppose that the following assumptions are satisfied:

(i) $\phi_{\eta}=0$,

(ii) $\phi_{b} \leq 0$

(iii) $u \tau \leq 0$,

(iv) $R_{j}=\bar{R}$ for $j=1, \ldots, n_{s}$.

Then, any smooth solution of (4.39) satisfies the entropy inequality

$$
\partial_{t}\left(\bar{\eta}(W)+\frac{g}{2} \bar{R} H^{2}\right)+\partial_{x} G(W) \leq 0,
$$

where $\eta$ and $G$ are given by (4.41) and (4.42) respectively.

\section{Proof.}

The following equality can be easily verified by using (iv).

$$
-\frac{g}{2} h R_{c} \phi_{b}+\frac{g}{2} h \sum_{j=1}^{n_{s}} R_{j} \phi_{b}^{j}-g H \sum_{j=1}^{n_{s}} R_{j} \phi_{b}^{j}=\frac{g}{2} h \bar{R}(1-c) \phi_{b}-g H \bar{R} \phi_{b} .
$$


Using this equality and $4.39 \mathrm{~d}$ we may write (4.40) in the form

$$
\partial_{t}\left(\eta(W)+g \bar{R} \frac{H^{2}}{2}\right)+\partial_{x}(G(W))=\left(\frac{u^{2}}{2}+g\left(R_{0}(h-H)+\frac{R_{c} h}{2}\right)\right) \phi_{\eta}+\frac{g}{2} h \bar{R}(1-c) \phi_{b}+u \tau,
$$

and the result follows.

Remark 4.1. As the source term $\phi_{b}$ is given by

$$
\phi_{b}=\sum_{j=1}^{n_{s}} v_{s_{j}}\left(p_{j} E_{s_{j}}-c_{b_{j}}\right)
$$

$\phi_{b} \leq 0$ as long as $c_{b_{j}} \geq p_{j} E_{s_{j}}$ i.e., if the deposition predominates over erosion.

Given the particular definition of $E_{s_{j}}$ in (2.9), this is the case in many physical situations.

\section{$5 \quad$ Numerical scheme}

As usual, we consider a set of computing cells $I_{i}=\left[x_{i-1 / 2}, x_{i+1 / 2}\right], i \in \mathbb{Z}$. For the sake of simplicity, we assume that these cells have a constant size $\triangle x$ and that $x_{i+1 / 2}=i \triangle x . x_{i}=(i-1 / 2) \triangle x$ is the center of the cell $I_{i}$. Let $\triangle t$ be the time step and $t^{n}=n \triangle t$.

We denote by $W_{i}^{n}$ the approximation of the cell averages of the exact solution

$$
W_{i}^{n} \cong \frac{1}{\triangle x} \int_{x_{i-1 / 2}}^{x_{i+1 / 2}} W\left(x, t^{n}\right) d x .
$$

Given the approximations $\left(W_{i}^{n}\right)$ at time $t_{n}$, we compute the approximations at time $t^{n+1}$ using a two-step algorithm.

First we solve

$$
\left\{\begin{array}{l}
\partial_{t} W+\partial_{x} \widetilde{F}(W)=B(W) \partial_{x} H \\
W\left(x, t=t^{n}\right)=W_{i}^{n} \text { for } x \in I_{i}
\end{array}\right.
$$

Let $W_{i}^{n+1 / 2}$ be the approximations of the cell averages of the solution of this problem. We define

$$
W_{i}^{n+1}=W_{i}^{n+1 / 2}+\triangle t S\left(W_{i}^{n}\right) .
$$

In order to solve (5.2), we introduce the theory described in [22], [21]. We briefly describe thereafter the application of the theory to the system considered here.

\subsection{Roe scheme adapted to system (5.2)}

System (5.2) may be written under the form

$$
\frac{\partial W}{\partial t}+\mathcal{A}(W) \frac{\partial W}{\partial x}=0, \quad x \in \mathbb{R}, t>0 .
$$

with $\mathcal{A}(W)$ defined by (4.4). This matrix has the following block structure

$$
\mathcal{A}(W)=\left[\begin{array}{c|c}
\mathcal{J}(U) & -b(U) \\
\hline-\xi \nabla q_{b} & 0
\end{array}\right]
$$

where $U=\left(h, h u, h c_{1}, \ldots, h c_{n_{s}}\right)^{t}, \mathcal{J}(U)=\nabla_{U} F, F(U)=\left(h u, h u^{2}+g / 2\left(R_{0}+R_{c}\right) h^{2}, h c_{1} u, \ldots, h c_{n_{s}} u\right)^{t}$ and $b(U)=\left(0,-g\left(R_{0} h+R_{c} h\right), 0, \ldots, 0\right)^{t}$.

Only the cases $q_{b}=0$ or $q_{b}$ given by Grass model will be considered here.

Observe that system (5.4) contains a non-conservative product $\mathcal{A}(W) \cdot W_{x}$ which, in general, does not make sense within the framework of the theory of distributions. After the theory developed by 
Dal Masso, LeFloch, and Murat [9], a rigorous definition of weak solutions can be performed using a family of paths. We refer to [9], [22] and [21] for further details.

In [24] a generalization of Roe methods to systems of the form (5.4) was introduced. These methods are based on the following general definition of a Roe linearization:

Definition 5.1. Given a family of paths $\Psi$, a matrix function $\mathcal{A}_{\Psi}: \Omega \times \Omega \rightarrow \mathcal{M}_{N}(\mathbb{R})$ is called a Roe linearization if it satisfies:

- for any $W_{L}, W_{R} \in \Omega, \mathcal{A}_{\Psi}\left(W_{L}, W_{R}\right)$ has $N$ real eigenvalues;

- $\mathcal{A}_{\Psi}(W, W)=\mathcal{A}(W)$, for all $W \in \Omega$;

- for any $W_{L}, W_{R} \in \Omega$ :

$$
\mathcal{A}_{\Psi}\left(W_{L}, W_{R}\right) \cdot\left(W_{R}-W_{L}\right)=\int_{0}^{1} \mathcal{A}\left(\Psi\left(s ; W_{L}, W_{R}\right)\right) \frac{\partial \Psi}{\partial s}\left(s ; W_{L}, W_{R}\right) d s .
$$

A family of paths is a Lipschitz continuous function $\Psi:[0,1] \times \Omega \times \Omega \rightarrow \Omega$ which must satisfy some regularity conditions, (see [9] for the details) and $\left.\Psi\left(0, W_{l}, W_{r}\right)=W_{l}, \Psi\left(1, W_{l}, W_{r}\right)=W_{r}\right)$, $\Psi(s, W, W)=W$.

Once a Roe linearization is given, Roe schemes can be generalized for systems of the form (5.4). We refer to [22] for further details. The scheme can be written in the form

$$
W_{i}^{n+1}=W_{i}^{n}-\frac{\Delta t}{\Delta x}\left(\mathcal{A}_{i-1 / 2}^{+} \cdot\left(W_{i}^{n}-W_{i-1}^{n}\right)+\mathcal{A}_{i+1 / 2}^{-} \cdot\left(W_{i+1}^{n}-W_{i}^{n}\right)\right),
$$

where

$$
\mathcal{L}_{i+1 / 2}^{ \pm}=\left[\begin{array}{ccc}
\left(\lambda_{1}^{i+1 / 2}\right)^{ \pm} & & 0 \\
& \ddots & \\
0 & & \left(\lambda_{N}^{i+1 / 2}\right)^{ \pm}
\end{array}\right], \quad \mathcal{A}_{i+1 / 2}^{ \pm}=\mathcal{K}_{i+1 / 2} \mathcal{L}_{i+1 / 2}^{ \pm} \mathcal{K}_{i+1 / 2}^{-1},
$$

$\lambda_{1}^{i+1 / 2}, \ldots, \lambda_{N}^{i+1 / 2}$ being the eigenvalues of $\mathcal{A}_{i+1 / 2}$ and $\mathcal{K}_{i+1 / 2}$ a matrix whose columns form a basis of associated eigenvectors.

We consider here the canonical choice of the family of paths

$$
\Psi\left(s ; W_{L}, W_{R}\right)=W_{L}+s\left(W_{R}-W_{L}\right) .
$$

In order to build a Roe linearization based on the family of paths (5.9), it is natural to look for matrices with the same structure of $\mathcal{A}(W)$ :

$$
\mathcal{A}_{i+1 / 2}=\left[\begin{array}{c|c}
\mathcal{J}_{i+1 / 2} & -b_{i+1 / 2} \\
\hline-\xi q_{i+1 / 2} & 0
\end{array}\right]
$$

For Roe matrices with this structure, (5.6) can be rewritten as follows:

$$
\begin{gathered}
\mathcal{J}_{i+1 / 2}\left(U_{i+1}-U_{i}\right)-\left(H_{i+1}-H_{i}\right) b_{i+1 / 2}=F\left(U_{i+1}\right)-F\left(U_{i}\right)-b_{\Psi}\left(W_{i}, W_{i+1}\right) \\
q_{i+1 / 2}\left(U_{i+1}-U_{i}\right)=q_{b}\left(U_{i+1}\right)-q_{b}\left(U_{i}\right),
\end{gathered}
$$

where

$$
b_{\Psi}\left(W_{i}, W_{i+1}\right)=\left(0, \int_{0}^{1} g\left(R_{0} \Psi_{1}\left(s, W_{i}, W_{i+1}\right)+\sum_{j=1}^{n_{s}} R_{j} \Psi_{j+2}\left(s, W_{i}, W_{i+1}\right)\right) \frac{\partial \Psi_{2}}{\partial s} d s, 0, \ldots, 0\right)^{t} .
$$


In the particular case that $q_{b}$ is given by Grass model, properties (5.11)-(5.12) are satisfied if we define:

$$
\begin{aligned}
& \mathcal{J}_{i+1 / 2}=\left(\begin{array}{ccccc}
0 & 1 & 0 & \ldots & 0 \\
g\left(R_{0} h_{i+1 / 2}+\frac{1}{2} \gamma_{i+1 / 2}\right)-\left(u_{i+1 / 2}\right)^{2} & 2 u_{i+1 / 2} & \frac{g}{2} R_{1} h_{i+1 / 2} & \ldots & \frac{g}{2} R_{n_{s}} h_{i+1 / 2} \\
-\left(c_{1}\right)_{i+1 / 2} u_{i+1 / 2} & \left(c_{1}\right)_{i+1 / 2} & u_{i+1 / 2} & \ddots & 0 \\
\ldots & \ldots & \ldots & \ldots & \ldots \\
-\left(c_{n_{s}}\right)_{i+1 / 2} & \left(c_{n_{s}}\right)_{i+1 / 2} & 0 & \ldots & u_{i+1 / 2}
\end{array}\right), \\
& -\xi q_{i+1 / 2}=\left(\begin{array}{lllll}
u_{i+1 / 2} d_{i+1 / 2} & -d_{i+1 / 2} & 0 & \ldots & 0
\end{array}\right), \\
& b_{i+1 / 2}=\left(\begin{array}{c}
0 \\
g\left(R_{0} h_{i+1 / 2}+\gamma_{i+1 / 2}\right) \\
0 \\
\cdots
\end{array}\right)
\end{aligned}
$$

where

$$
\begin{gathered}
h_{i+1 / 2}=\frac{h_{i}+h_{i+1}}{2}, \quad u_{i+1 / 2}=\frac{\sqrt{h_{i}} u_{i}+\sqrt{h_{i+1}} u_{i+1}}{\sqrt{h_{i}}+\sqrt{h_{i+1}}}, \\
\left(c_{j}\right)_{i+1 / 2}=\frac{\sqrt{h_{i}}\left(c_{j}\right)_{i}+\sqrt{h_{i+1}}\left(c_{j}\right)_{i+1}}{\sqrt{h_{i}}+\sqrt{h_{i+1}}}, \quad \gamma_{i+1 / 2}=\frac{\sum_{j=1}^{n_{s}} R_{j}\left(\left(h c_{j}\right)_{i}+\left(h c_{j}\right)_{i+1}\right)}{2}, \\
d_{i+1 / 2}=\frac{A_{g} \xi\left(\sqrt{h_{i}}+\sqrt{h_{i+1}}\right)}{\sqrt{h_{i}} h_{i+1}+\sqrt{h_{i+1}} h_{i}} \sum_{k=0}^{m_{g}-1}\left(u_{i+1}\right)^{k}\left(u_{i}\right)^{m_{g}-(k+1)} .
\end{gathered}
$$

The eigenstructure of matrices (5.10) is given by lemma 4.2, and thus $\mathcal{A}_{i+1 / 2}$ is a Roe matrix.

Remark 5.1. If we neglect the solid transport flow $\left(q_{b}=0\right)$, the system reduces to a system of conservation laws with a source term. Thus, following [22], we have

$$
\mathcal{A}_{i+1 / 2}^{ \pm}=\left[\begin{array}{c|c}
\mathcal{J}_{i+1 / 2}^{ \pm} & -\mathcal{J}_{i+1 / 2}^{ \pm} \mathcal{J}_{i+1 / 2}^{-1} b_{i+1 / 2} \\
\hline 0 & 0
\end{array}\right]
$$

and the scheme (5.7) may be rewritten under the form

$$
\begin{aligned}
U_{i}^{n+1}=U_{i}^{n} & -\frac{\triangle t}{\triangle x}\left[P_{i-1 / 2}^{+}\left(\mathcal{J}_{i-1 / 2}\left(U_{i}-U_{i-1}\right)-b_{i-1 / 2}\left(H_{i}-H_{i-1}\right)\right)\right. \\
& \left.+P_{i+1 / 2}^{-}\left(\mathcal{J}_{i+1 / 2}\left(U_{i+1}-U_{i}\right)-b_{i+1 / 2}\left(H_{i+1}-H_{i}\right)\right)\right]
\end{aligned}
$$

where

$$
P_{i+1 / 2}^{ \pm}=\frac{1}{2}\left(I d \pm\left|\mathcal{J}_{i+1 / 2}\right| \mathcal{J}_{i+1 / 2}^{-1}\right)
$$

\subsection{The non-hyperbolic case}

From Lemma 4.2 , if $R_{0} h_{i+1 / 2}+\frac{1}{2} \gamma_{i+1 / 2}+\frac{1}{2} \sum_{j=1}^{n_{s}}\left(R_{j} c_{j}\right)_{i+1 / 2} h_{i+1 / 2}>0$ and $d_{i+1 / 2}$ is sufficiently small, $\mathcal{A}_{i+1 / 2}$ has $n_{s}+3$ real eigenvalues that can be approached by

$$
\begin{gathered}
\lambda_{1} \simeq u_{i+1 / 2}-\sqrt{g\left(R_{0} h_{i+1 / 2}+\frac{\gamma_{i+1 / 2}}{2}+\frac{1}{2}\left(R_{c}\right)_{i+1 / 2} h_{i+1 / 2}\right)}, \\
\lambda_{2} \simeq u_{i+1 / 2}+\sqrt{g\left(R_{0} h_{i+1 / 2}+\frac{\gamma_{i+1 / 2}}{2}+\frac{1}{2}\left(R_{c}\right)_{i+1 / 2} h_{i+1 / 2}\right)}, \\
\lambda_{2+j}=u_{i+1 / 2}, j=1, \ldots, n_{s}\left(\text { multiplicity } n_{s}\right), \\
\lambda_{n_{s}+3} \simeq 0 .
\end{gathered}
$$


As $R_{0}<0, \lambda_{1}$ and $\lambda_{2}$ may become complex when $\frac{1}{2} \gamma_{i+1 / 2}+\frac{1}{2}\left(R_{c}\right)_{i+1 / 2} h_{i+1 / 2}$ is small. Indeed, this situation may arise near the plume front or when most of the sediment has been deposited. In this situations the density of the turbidity current is lower than that of the ambient water an thus it should go upwards. Therefore, the model is no longer valid in such situations. Nevertheless, one would like to compute, for example, numerical simulations of dam-break type, where eigenvalues may become complex near the front but the model makes sense far from the front. One way to do so is to set $\rho_{0}=\rho_{w}$ if $\mathcal{A}_{i+1 / 2}$ has complex eigenvalues and to neglect the pressure terms. Thus, whenever $\mathcal{A}_{i+1 / 2}$ given by $(5.10)$, (5.14)-(5.16) has complex eigenvalues, we replace locally the scheme by an up-winding method for the flux $\widetilde{F}$ without pressure terms. More explicitly, if the Roe matrix has complex eigenvalues at the inter-cell $x_{i_{0}+1 / 2}$, then the terms $\mathcal{A}_{i_{0}+1 / 2}^{ \pm}\left(W_{i_{0}+1}-W_{i_{0}}\right)$ in the scheme (5.7) are replaced by $D_{i_{0}+1 / 2}^{ \pm}$where

$$
\begin{array}{ll}
D_{i_{0}+1 / 2}^{-}=0, \quad D_{i_{0}+1 / 2}^{+}=\widetilde{F}^{*}\left(W_{i_{0}+1}\right)-\widetilde{F}^{*}\left(W_{i_{0}}\right) & \text { if } u_{i_{0}+1 / 2}>0 \\
D_{i_{0}+1 / 2}^{-}=\widetilde{F}^{*}\left(W_{i_{0}+1}\right)-\widetilde{F}^{*}\left(W_{i_{0}}\right), \quad D_{i_{0}+1 / 2}^{+}=0 & \text { if } u_{i_{0}+1 / 2}<0
\end{array}
$$

and

$$
\widetilde{F}^{*}(W)=\left(h u, h u^{2}, h c_{1} u, \ldots, h c_{n_{s}} u,-\xi q_{b}\right)^{t}
$$

\subsection{Addition of a non-erodible bed}

Usually, the bottom is formed by a thin layer of sediments that may be eroded or transported by the plume that lays over a rigid bottom which is not erodible. In that case, an initial function $M_{0}(x)$ must be given that establishes the depth for the non-erodible bottom (see Figure 2). Moreover, in some cases a fraction of the deposited sediment may solidify and become part of the non-erodible bottom so that $M_{0}$ could depend on time.

In this cases, the source terms $\left(\phi_{b}^{j}\right)^{n}=v_{s_{j}}\left(p_{j}^{n} E_{s_{j}}^{n}-c_{b_{j}}^{n}\right)$ have to be limited so that the amount of eroded sediment do not exceed the total mass of the erodible layer. Thus, for each cell $I_{i}$ whenever $\left(\phi_{b}^{j}\right)^{n}>0$, this source term is replaced by the modified expression

$$
\left(\widehat{\phi}_{b}^{j}\right)^{n}= \begin{cases}\left(\phi_{b}^{j}\right)^{n}, & \text { if } p_{j}^{n}\left(M_{0}-H^{n}\right)-\xi \triangle t\left(\phi_{b}^{j}\right)^{n} \geq 0 \\ p_{j}^{n}\left(M_{0}-H^{n}\right)(\xi \triangle t)^{-1}, & \text { otherwise. }\end{cases}
$$

The volume fraction of sediments at the bottom, $p_{i}^{n+1}$ is given by

$$
p_{j}^{n+1}\left(M_{0}-H^{n+1}\right)=p_{j}^{n}\left(M_{0}-H^{n}\right)-\xi \triangle t\left(\widehat{\phi}_{b}^{j}\right)^{n} \geq 0
$$

for each cell $I_{i}$. Note that (5.30) assures the positivity of $p_{j}^{n+1}$.

\section{Extension to 2D}

The model (2.17) can be easily extended to the 2D case. Following the same procedure of Section 3 adapted to the $2 \mathrm{D}$ case, one can obtain

$$
\left\{\begin{array}{l}
\partial_{t} h+\partial_{x}(h u)+\partial_{y}(h v)=\phi_{\eta}+\phi_{b}, \\
\partial_{t}(h u)+\partial_{x}\left(h u^{2}+g\left(R_{0}+R_{c}\right) \frac{h^{2}}{2}\right)+\partial_{y}(h u v)=g\left(R_{0}+R_{c}\right) h \partial_{x} H+u \phi_{\eta}+\frac{u}{2} \phi_{b}+\tau_{u}, \\
\partial_{t}(h v)+\partial_{x}(h u v)+\partial_{y}\left(h v^{2}+g\left(R_{0}+R_{c}\right) \frac{h^{2}}{2}\right)=g\left(R_{0}+R_{c}\right) h \partial_{y} H+v \phi_{\eta}+\frac{v}{2} \phi_{b}+\tau_{v}, \\
\partial_{t}\left(h c_{j}\right)+\partial_{x}\left(h u c_{j}\right)+\partial_{y}\left(h v c_{j}\right)=\phi_{b}^{j}, \text { for } j=1, \ldots, n_{s} \\
\partial_{t} H-\xi \partial_{x} q_{b}^{x}-\xi \partial_{y} q_{b}^{y}=\xi \phi_{b},
\end{array}\right.
$$




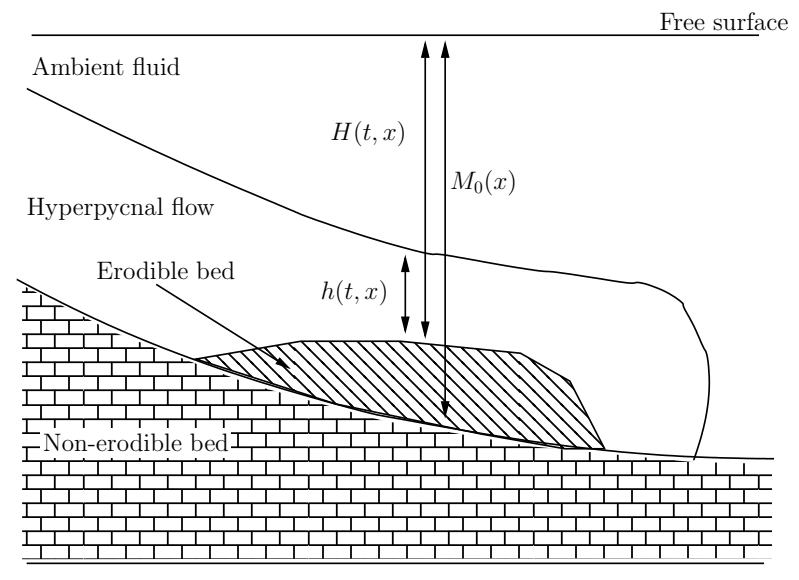

Figure 2: Sketch of hyperpycnal flow over a non erodible bed

where $h$ is the current thickness; $\vec{u}=(u, v)$ is the depth-averaged velocity; $c_{j}$ for $j=1, \ldots, n_{s}$ represents the vertically averaged volume concentration of the $j$ th sediment; and $H$ is the bottom depth from a fixed level.

We shall adopt the same notation as in Section 2 adapting the source terms to the 2D framework. So, the expression of $\phi_{\eta}$ is replaced by

$$
\phi_{\eta}=E_{w}\|\vec{u}\|,
$$

while the definition of $\phi_{b}^{j}$ remains essentially the same but replacing $|u|$ by $\|\vec{u}\|$ in (2.10). We shall denote

$$
\tau_{u}=-(1+\alpha) c_{D} u\|\vec{u}\|, \quad \tau_{v}=-(1+\alpha) c_{D} v\|\vec{u}\| .
$$

$\left(q_{b}^{x}, q_{b}^{y}\right)$ is the corresponding $2 \mathrm{D}$ solid transport flux which, in the case of Grass model, corresponds to

$$
q_{b}^{x}=A_{g} u\|\vec{u}\|^{m_{g}-1}, \quad q_{b}^{y}=A_{g} v\|\vec{u}\|^{m_{g}-1}, \quad 1 \leq m_{g} \leq 4,
$$

The system may be rewritten in the form:

$$
\partial_{t} W+\partial_{x} \widetilde{F}_{1}(W)+\partial_{y} \widetilde{F}_{2}(W)=B_{1}(W) \partial_{x} W+B_{2}(W) \partial_{y} W+S(W),
$$

where $W=\left(h, h u, h v, h c_{1}, \ldots, h c_{n_{s}}, H\right)^{t}$ and

$$
\begin{aligned}
& \widetilde{F}_{1}(W)=\left(h u, h u^{2}+g / 2\left(R_{0}+R_{c}\right) h^{2}, h u v, h c_{1} u, \ldots, h c_{n_{s}} u,-\xi q_{b}^{x}\right)^{t}, \\
& \widetilde{F}_{2}(W)=\left(h v, h u v, h v^{2}+g / 2\left(R_{0}+R_{c}\right) h^{2}, h c_{1} v, \ldots, h c_{n_{s}} v,-\xi q_{b}^{y}\right)^{t}, \\
& B_{1}(W)=g\left(R_{0}+R_{c}\right) h e_{2, n_{s}+4}, \\
& B_{2}(W)=g\left(R_{0}+R_{c}\right) h e_{3, n_{s}+4}
\end{aligned}
$$

and

$$
S(W)=\left(\begin{array}{c}
E_{w}\|\vec{u}\|+\sum_{j=1}^{n_{s}} v_{s_{j}}\left(p_{j} E_{s_{j}}-c_{b_{j}}\right) \\
E_{w} u\|\vec{u}\|+\frac{u}{2} \sum_{j s}^{n_{s}=1} v_{s_{j}}\left(p_{j} E_{s_{j}}-c_{b_{j}}\right)-(1+\alpha) c_{D} u\|\vec{u}\| \\
E_{w} v\|\vec{u}\|+\frac{v}{2} \sum_{j=1}^{n_{s}} v_{s_{j}}\left(p_{j} E_{s_{j}}-c_{b_{j}}\right)-(1+\alpha) c_{D} v\|\vec{u}\| \\
v_{s_{1}}\left(p_{1} E_{s_{1}}-c_{b_{1}}\right) \\
\ldots \\
v_{s_{n_{s}}}\left(p_{n_{s}} E_{s_{n_{s}}}-c_{b_{n_{s}}}\right) \\
\xi \sum_{j=1}^{n_{s}} v_{s_{j}}\left(p_{j} E_{s_{j}}-c_{b_{j}}\right)
\end{array}\right)
$$

Here $e_{r, s}=\left(\delta_{i r} \delta_{j s}\right)_{i=1, j=1}^{n_{s}+4, n_{s}+4}$ is the canonical basis of square matrices of order $n_{s}+4$. The problem may also be written in the more general form 


$$
\partial_{t} W+\mathcal{A}_{1}(W) \partial_{x} W+\mathcal{A}_{2}(W) \partial_{y} W=S(W)
$$

where $\mathcal{A}_{k}=\nabla \widetilde{F}_{k}(W)-B_{k}(W), \quad k=1,2$.

Some easy calculations allow us to prove the following property:

Lemma 6.1. System (6.1) is invariant under rotations. More explicitly, suppose any unitary vector $\vec{n}=\left(n_{x}, n_{y}\right)$ and define

$$
R_{\vec{n}}=\left[\begin{array}{c|cc|c}
1 & 0 & 0 & 0 \\
\hline 0 & n_{x} & n_{y} & 0 \\
0 & -n_{y} & n_{x} & 0 \\
\hline 0 & 0 & 0 & I d
\end{array}\right]
$$

Then the following relations hold

$$
\begin{aligned}
& \widetilde{F}_{\vec{n}}(W):=n_{x} \widetilde{F}_{1}(W)+n_{y} \widetilde{F}_{2}(W)=R_{\vec{n}}^{-1} \widetilde{F}_{1}\left(R_{\vec{n}} W\right), \\
& B_{\vec{n}}(W):=n_{x} B_{1}(W)+n_{y} B_{2}(W)=R_{\vec{n}}^{-1} B_{1}\left(R_{\vec{n}} W\right) R_{\vec{n}}, \\
& \mathcal{A}_{\vec{n}}(W):=n_{x} \mathcal{A}_{1}+n_{y} \mathcal{A}_{2}=R_{\vec{n}}^{-1} \mathcal{A}_{1}\left(R_{\vec{n}} W\right) R_{\vec{n}} .
\end{aligned}
$$

In order to solve (6.11), the computational domain is divided into discretization cells or finite volumes, $V_{i}$ and we proceed as it was done in Section 5 by solving first

$$
\left\{\begin{array}{l}
\partial_{t} W+\partial_{x} \widetilde{F}_{1}(W)+\partial_{y} \widetilde{F}_{2}(W)=B_{1}(W) \partial_{x} H+B_{2}(W) \partial_{y} H \\
W\left(x, y, t=t^{n}\right)=W_{i}^{n} \text { for }(x, y) \in V_{i}
\end{array}\right.
$$

Then, let $W_{i}^{n+1 / 2}$ be the approximations of the cell averages of the solution of this problem. We define

$$
W_{i}^{n+1}=W_{i}^{n+1 / 2}+\triangle t S\left(W_{i}^{n}\right) .
$$

The following notation is considered: given a finite volume $V_{i}, \mathcal{N}_{i}$ is the set of indexes $j$ such that $V_{j}$ is a neighbor of $V_{i} ; E_{i j}$ is the common edge to two neighbor cells $V_{i}$ and $V_{j}$, and $\left|E_{i j}\right|$ represents its length; $\vec{n}_{i j}=\left(n_{i j, x}, n_{i j, y}\right)$ is the normal unit vector of the edge $E_{i j}$ pointing towards the cell $V_{j}$.

Following [7], [6], system (6.16) will be solved using a path-conservative Roe scheme which can be written under the form

$$
W_{i}^{n+1}=W_{i}^{n}-\frac{\triangle t}{\left|V_{i}\right|} \sum_{j \in \mathcal{N}_{i}}\left|E_{i j}\right| \mathcal{A}_{i j}^{-}\left(W_{j}^{n}-W_{i}^{n}\right),
$$

where $\mathcal{A}_{i, j}=\mathcal{A}\left(W_{i}, W_{j}, \vec{n}_{i j}\right)$ in now a Roe linearization of $n_{x} \mathcal{A}_{1}(W)+n_{y} \mathcal{A}_{2}(W)$. We refer to [7] and [6] for further details.

Thanks to Lemma 6.1, the definition of Roe matrix is an easy task by using the expression given in the $1 \mathrm{D}$ case. The eigenstructure of Roe matrix is also straightforward from Lemma 4.2.

\section{$7 \quad$ Numerical simulations}

\subsection{Comparison with laboratory experiments}

First, we compare the model with the data presented in [16] corresponding to a laboratory experiment using a 10-m-long, 0.2-m-wide and 0.5-m-deep flume with a 0.5-m-long gate box at the upstream end. Lock exchange experiments where studied with different bottom topographies and different sediment concentration at the gate box. For the experiment shown here, the initial water depth is $0.2 \mathrm{~m}$ at the gate box. The topography in the flume is given by Figure 3 .

Particle-driven density currents were generated by releasing suspensions, which were composed by siliceous, non-cohesive, sand to silt sized particles (density $2650 \mathrm{~kg} / \mathrm{m}^{3}$ ). The initial volume fraction of the particles was $2 \%$ and the solid transport of the bottom is neglected $\left(q_{b}=0\right)$. The sediments 


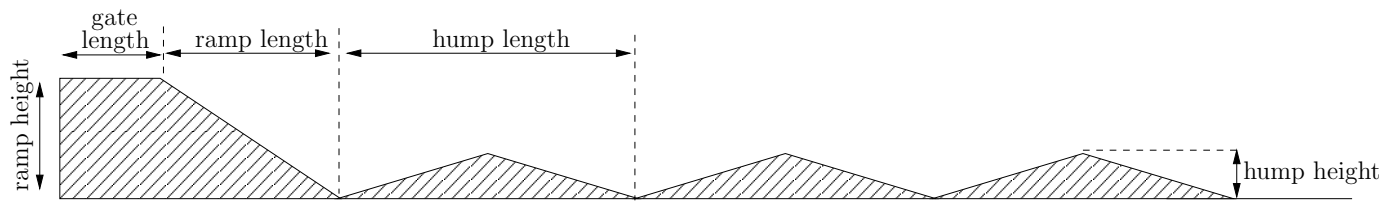

Figure 3: Comparison with laboratory experiments: Hump topography

are represented by a mixture of $20 \%$ of fine sand $\left(v_{s}=0.01 \mathrm{~m} / \mathrm{s}, \phi=3.0\right), 50 \%$ very fine sand $(v s=0.005 \mathrm{~m} / \mathrm{s}, \phi=3.5)$ and $30 \%$ of coarse silt $(v s=0.003 \mathrm{~m} / \mathrm{s}, \phi=4.0)$.

We show the simulations corresponding to experiments B5, B9, B11, C5, C7 and C13 in [16]. The corresponding parameters for each experiment are given in Table 1.

\begin{tabular}{cccccc}
\hline Experiment & $\begin{array}{c}\text { Ramp } \\
\text { height }\end{array}$ & $\begin{array}{c}\text { Ramp } \\
\text { length }\end{array}$ & $\begin{array}{c}\text { Number } \\
\text { of humps }\end{array}$ & $\begin{array}{c}\text { Hump } \\
\text { height }\end{array}$ & $\begin{array}{c}\text { Hump } \\
\text { length }\end{array}$ \\
\hline B5 & 0.1 & 1 & 0 & - & - \\
B9 & 0.1 & 2 & 0 & - & - \\
B11 & 0.05 & 1 & 0 & - & - \\
C5 & 0.1 & 1 & 3 & 0.036 & 1 \\
C7 & 0 & 1 & 3 & 0.036 & 1 \\
C13 & 0.1 & 1 & 3 & 0.036 & 2
\end{tabular}

Table 1: Experiment description

In general, the model predictions are comparable to the experimental results. In some cases, we remark that some disagreements arise, specially in the proximal area, where the model predicts less deposition than found in experimental results. This behaviour was also observed in the simulations shown in [16]. This is more likely due to the fact that the motion of the upper ambient layer has been neglected. When a turbidity current intrudes shallow water, an opposing current is generated in the ambient fluid, which reduces its forward momentum. In Figures 4, 5 and 6 we see that deposits decrease from a maximum at the beginning of the flume with a slight increase downstream of the slope break. This increase is sensible to the slope of the ramp. In the case of Figures 7, 8 and 9, the topography influence can be seen as an increase on upslope of the humps.

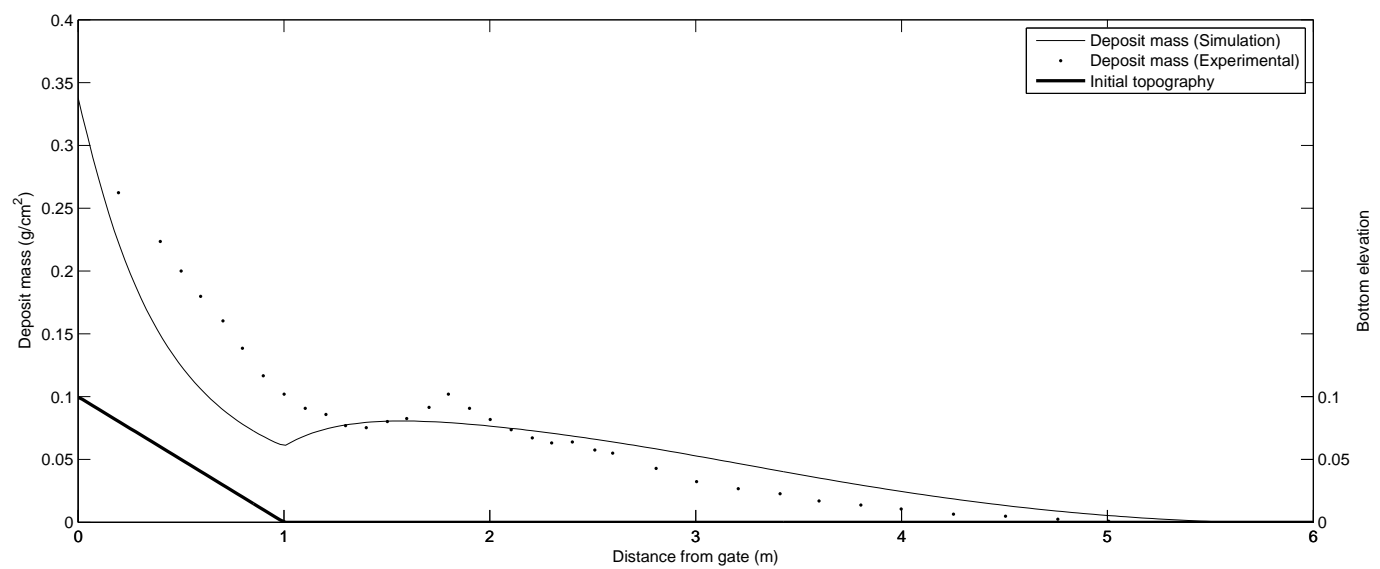

Figure 4: Comparison with laboratory experiments: Deposit distribution for experiment B5 


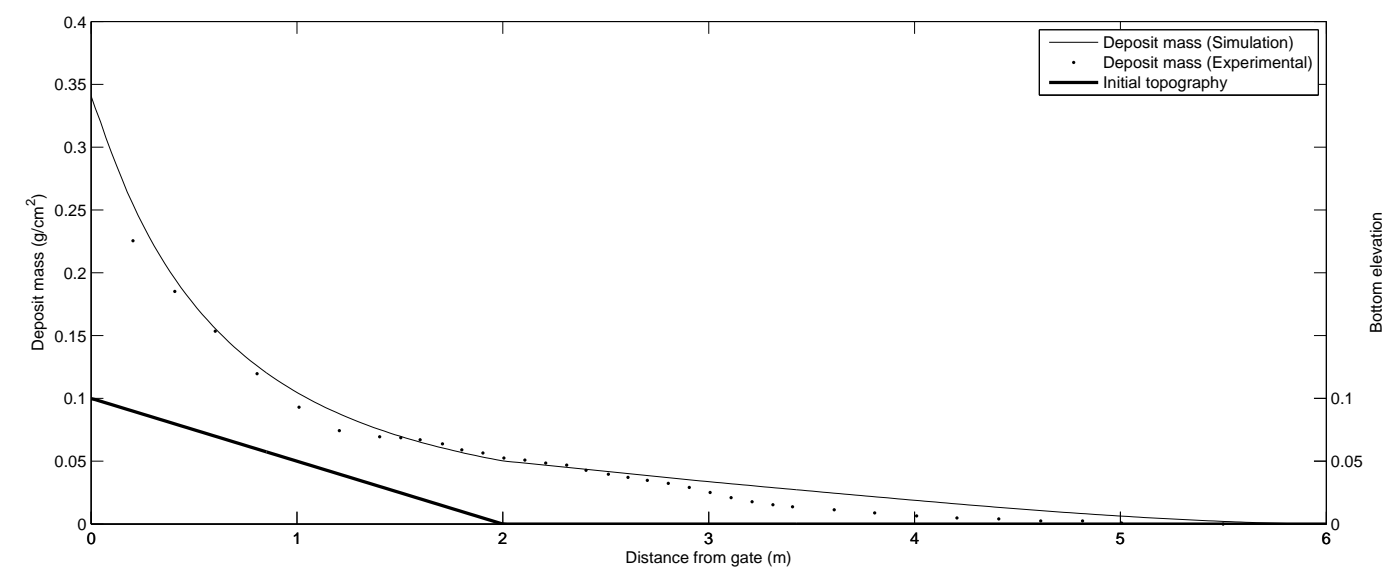

Figure 5: Comparison with laboratory experiments: Deposit distribution for experiment B9

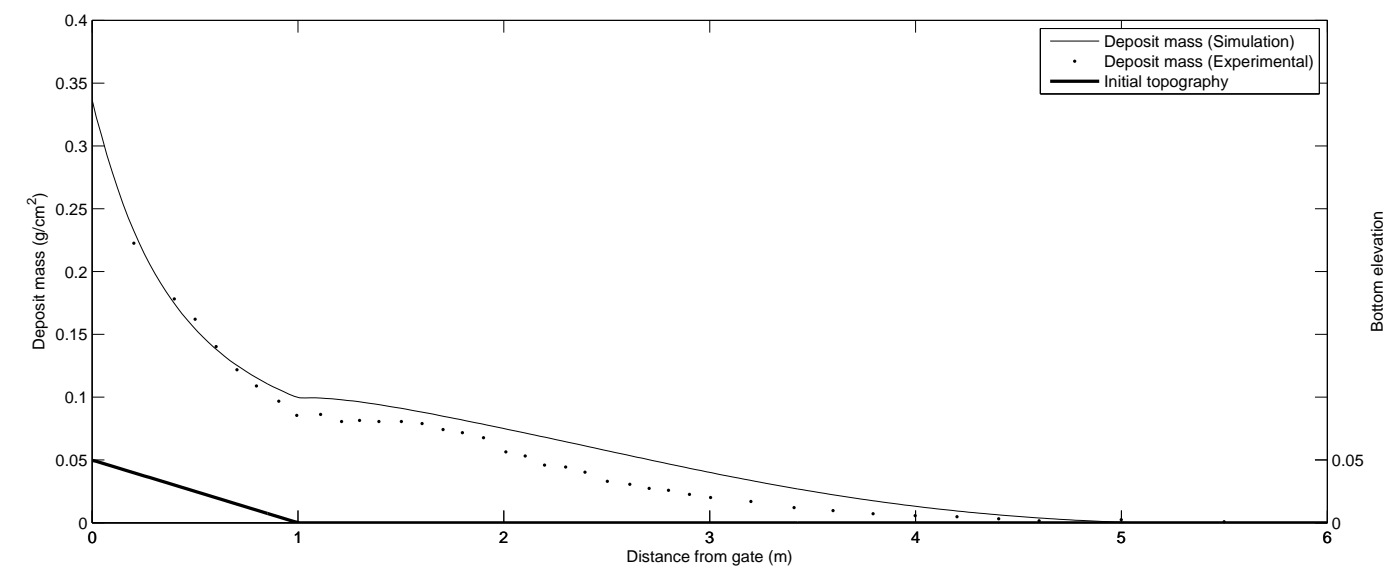

Figure 6: Comparison with laboratory experiments: Deposit distribution for experiment B11

\subsection{Successive turbidity currents over an initial flat bottom}

Inspired by the experiments shown in [17], we intend now to simulate spillover turbidity currents on submarine channel levees, that is a succession over a long time period of several turbidity currents. Following this idea, we generate successive turbidity currents over an initial $25 \mathrm{~m}$ long flat bottom. Each turbidity current is generated by supplying a suspension of sediments at the upstream end of the flow field. The mixture is composed by three sediment species with the same characteristics as the ones used on experiments given by Table 1 . The height is fixed to $0.5 \mathrm{~m}$ and the flux is $0.001 \mathrm{~m}^{2} / \mathrm{s}$. The supply duration is set at $4 s$ and the turbidity is let to evolve for $100 s$ before the next supply arrives. The volume of deposits is then converted to bed thickness so that the final bed after each supply is considered as the initial topography for the following and is supposed to solidify and to be non-erodible when the next plume arrives. In Figure 10 we represent the bottom evolution for this successive flow events. Each layer represents the resulting bottom after 500 flow events. We remark the appearance of wavy structures by repeated deposition of turbidities over a flat bottom as it would be expected in physical situations. 


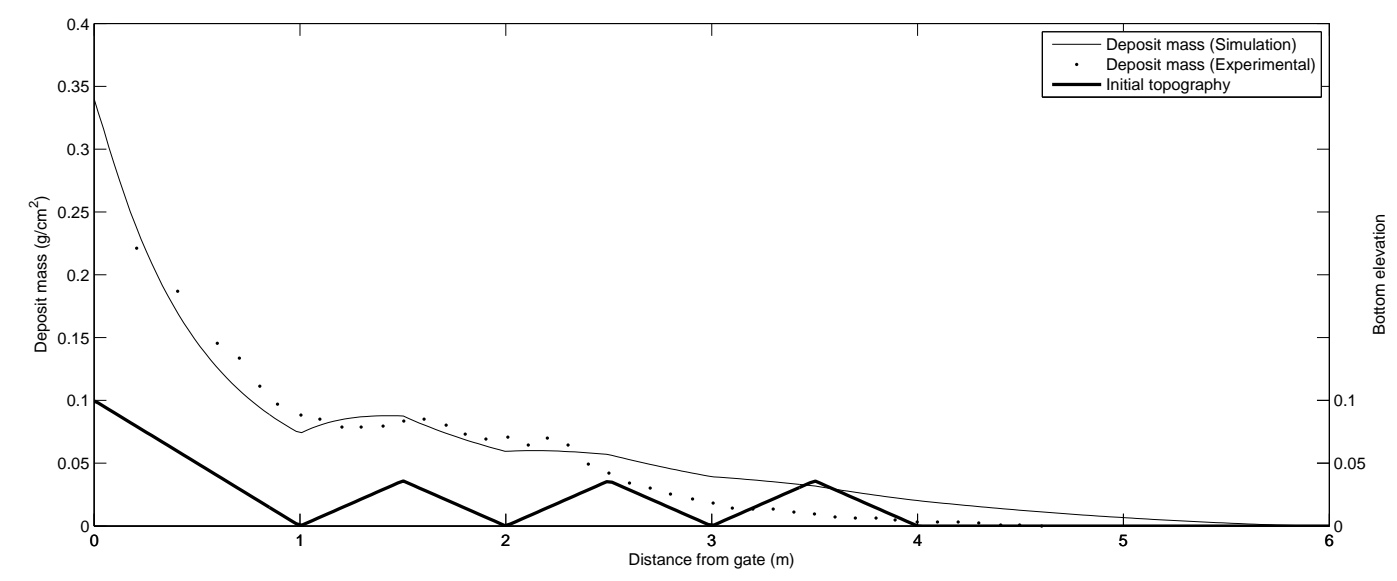

Figure 7: Comparison with laboratory experiments: Deposit distribution for experiment C5

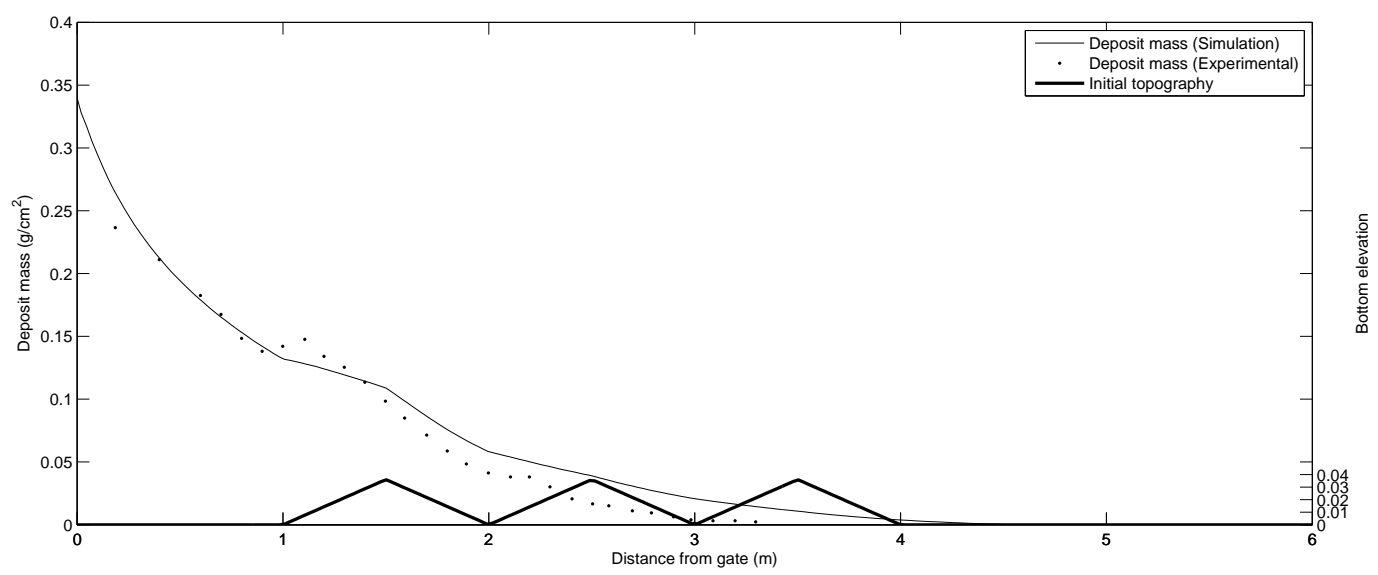

Figure 8: Comparison with laboratory experiments: Deposit distribution for experiment C7

\subsection{Solid transport of topography}

We shall now show the influence of solid transport on the topography. We consider the case of a river (assume $R_{0}=0$ ) over an initial topography given by

$$
H(x, t=0)= \begin{cases}0.1-0.04 \sin ^{2}(\pi(x-3) / 2), & \text { for } 2 \leq x \leq 5 \\ 0.1, & \text { elsewhere }\end{cases}
$$

This initial topography is assumed to be erodible up to the depth level 0.102 . The initial height is set to $h(x, t=0)=H(x, 0)$ and the initial velocity is such that $h u(x, t=0)=0.01$. We suppose that there is a suspension of siliceous particles (density $2650 \mathrm{~kg} / \mathrm{m}^{3}$ and $v s=0.002 \mathrm{~m} / \mathrm{s}$ ) with initial volume fraction of $1 \%$. At the upstream end, we set a supply with concentration $1 \%$ for the first $100 s$. Solid transport is considered using Grass model with $A_{g}=0.005$.

First, assume that there is no erosion of the topography and no deposition of particles, so that only solid transport is considered. Figure 11 shows the topography evolution.

Now, let us consider the same situation but adding erosion and deposition effects. The results are shown on Figure 12. 


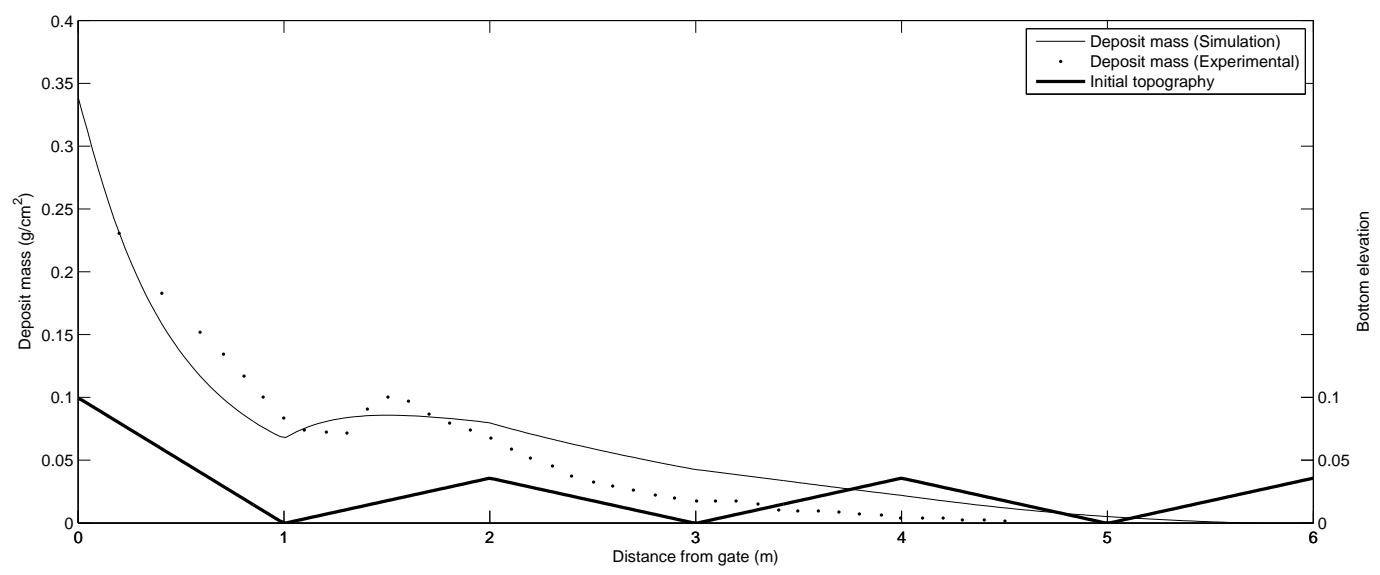

Figure 9: Comparison with laboratory experiments: Deposit distribution for experiment C13

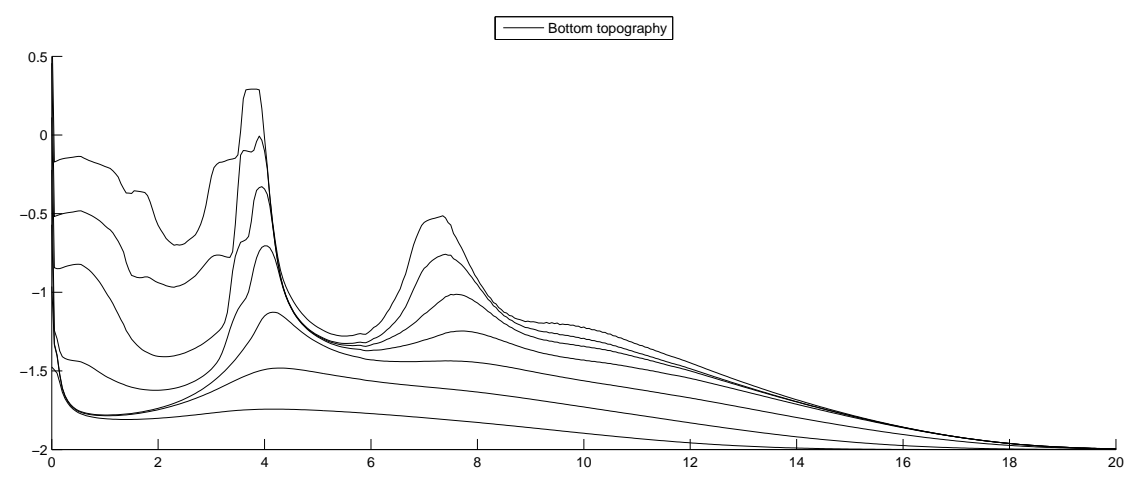

Figure 10: Successive turbidity currents over an initial flat bottom: Topography evolution. Each layer corresponds to 500 turbidity beds

\subsection{A 2D example}

As it has been said in Section 6, the model and numerical schemes can be easily extended to 2D turbidity currents. Here we shall show a test case related to experiment C5 in Section 7.1. We consider the domain given by Figure 13. A 0.5 -m-long by 1-m-wide gate box has been placed at the upstream end with a mixture of sediments with same characteristics as the ones used in Section 7.1. The initial height of the mixture is set to $0.2 \mathrm{~m}$ and we consider a hump topography like the one on Figure 14 with ramp and hump height and length corresponding to experiment C5 in Section 7.1. Figures 16-17 show the deposit density along the longitudinal and transversal sections on Figure 13. 


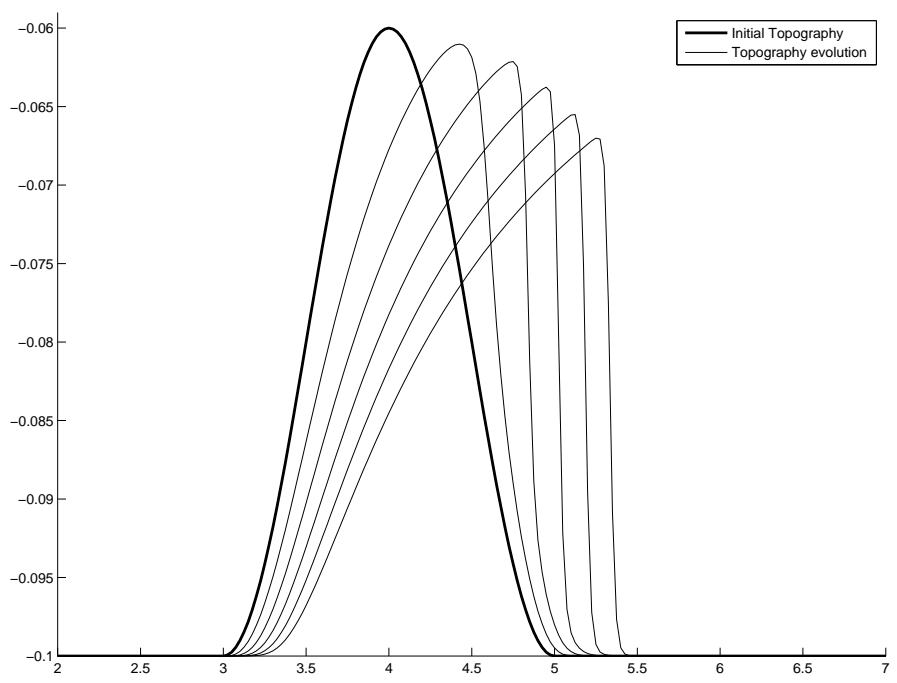

Figure 11: Solid transport of topography: Bottom evolution without erosion nor deposition of particles. Each layer represents $400 \mathrm{~s}$

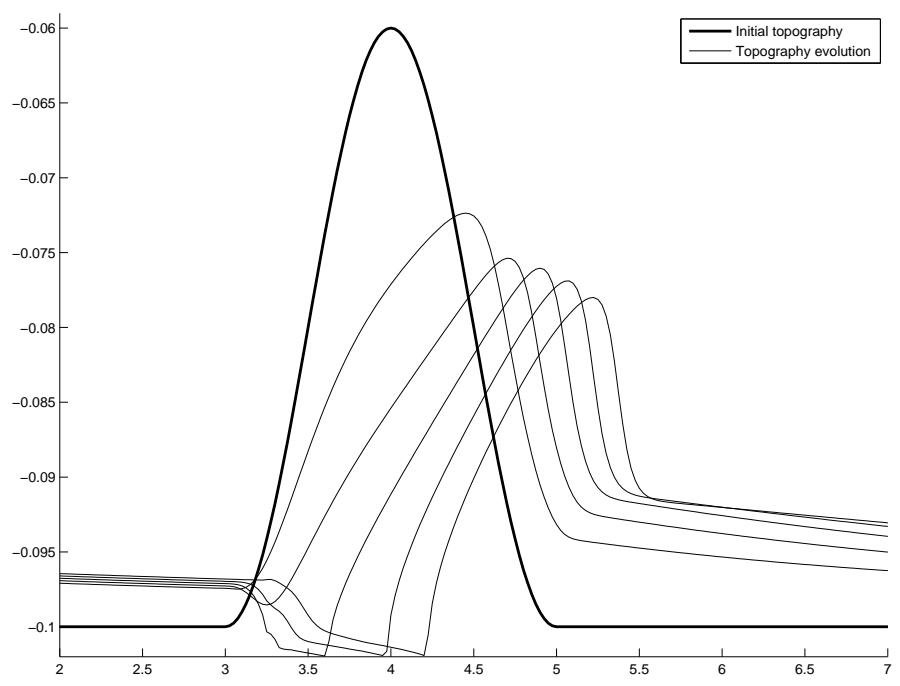

Figure 12: Solid transport of topography: Bottom evolution with erosion and deposition of particles. Each layer represents $400 \mathrm{~s}$ 


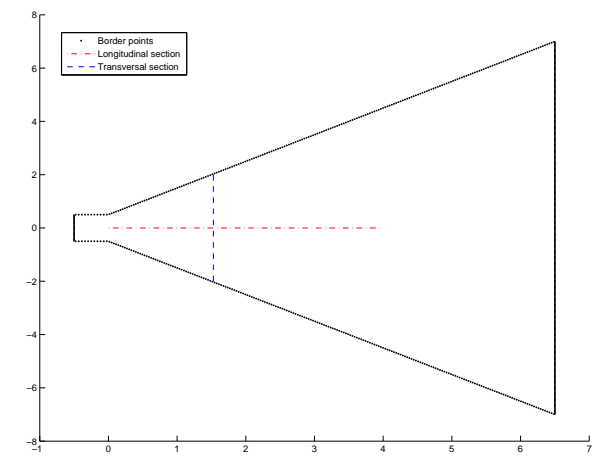

Figure 13: A 2D example: Domain and sections

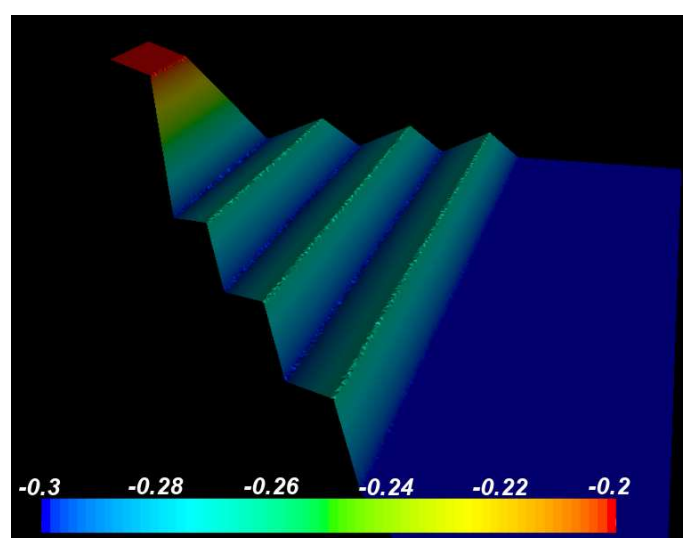

Figure 14: A 2D example: Bottom depth

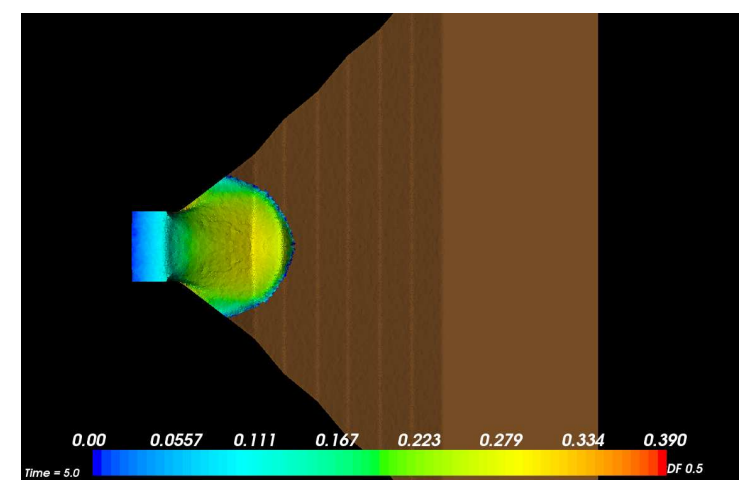

(a)

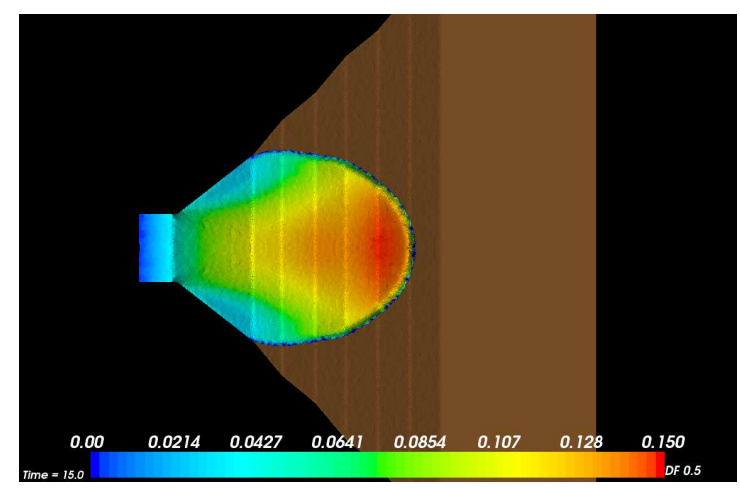

(c)

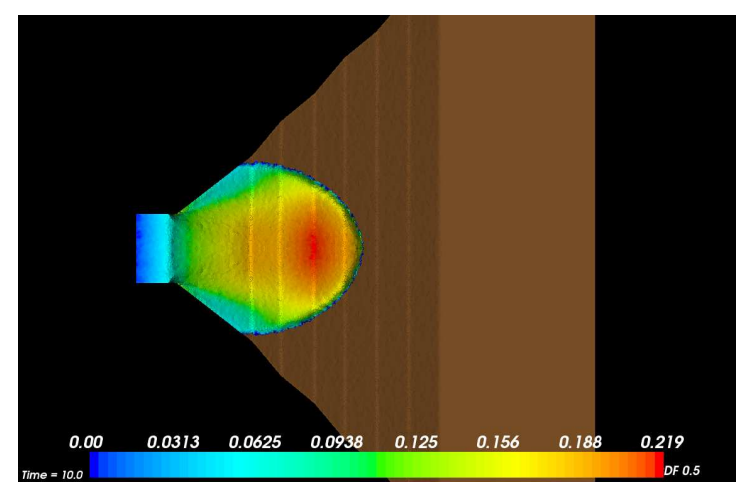

(b)

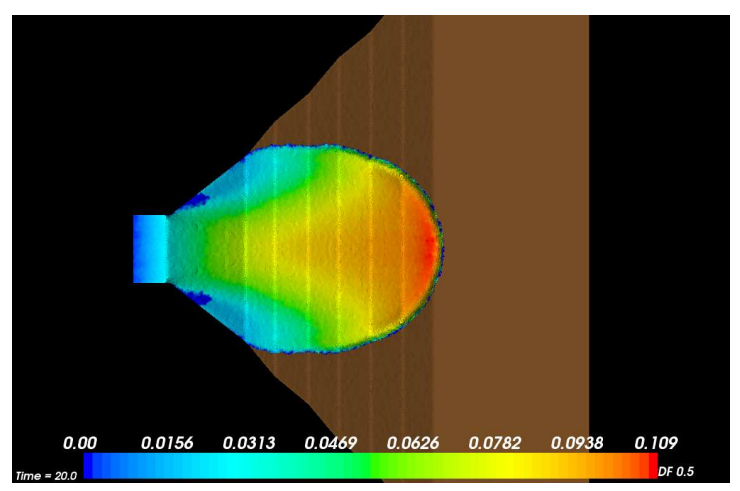

(d)

Figure 15: A 2D example: Evolution of turbidity current after release from the gate box. Color levels correspond to the modulus of velocity 


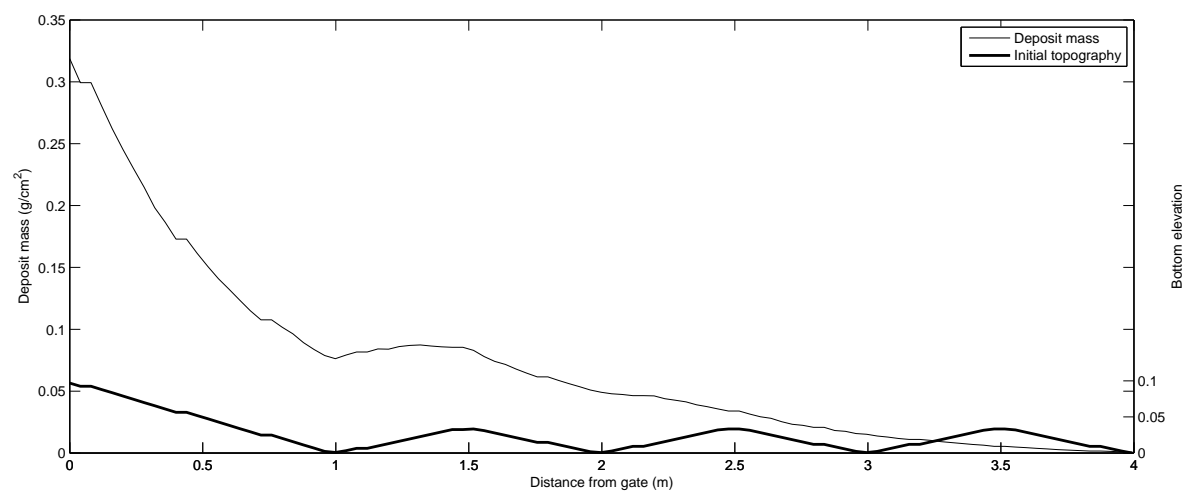

Figure 16: A 2D example: Deposit density. Longitudinal section

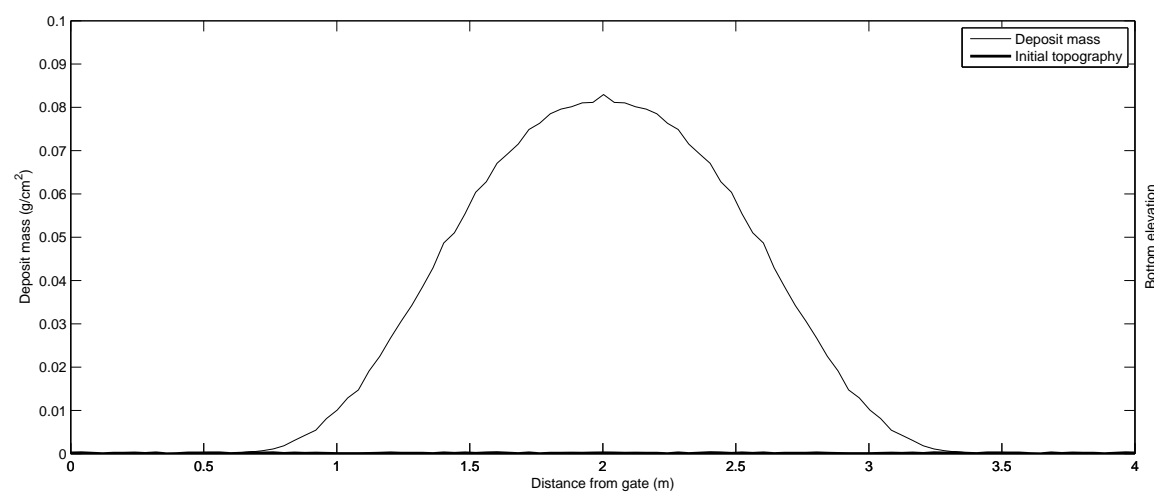

Figure 17: A 2D example: Deposit density. Transversal section 


\section{References}

[1] J. Alexander and S. Morris. Observations on experimental, nonchannelized, high-concentration turbidity currents and variations in deposits around obstacles. Journal of Sedimentary Research, 64:899-909, Oct. 1994.

[2] M. Altinaker, W. Graf, and E. Hopfinger. Flow structure in turbidity currents. Journal of Hydraulic Research, 34(5), 1996.

[3] R. T. Bonnecaze, H. E. Huppert, and J. R. Lister. Particle-driven gravity currents. Journal of Fluid Mechanics, 250:339-369, 1993.

[4] F. Bouchut, E. Fernández-Nieto, A. Mangeney, and P.-Y. Lagrée. On new erosion models of savage-hutter type for avalanches. To appear in Acta Mechanica.

[5] S. F. Bradford and N. D. Katopodes. Hydrodynamics of turbid underflows. i: Formulation and numerical analysis. Journal of Hydraulic Engineering, 125(10):1006-1015, 1999.

[6] M. Castro, E. Fernández-Nieto, A. Ferreiro, J. García-Rodríguez, and C. Parés. High order extensions of roe schemes for two-dimensional nonconservative hyperbolic systems. Journal of Scientific Computing.

[7] M. J. Castro, J. A. García-Rodríguez, J. M. González-Vida, and C. Parés. A parallel 2d finite volume scheme for solving systems of balance laws with nonconservative products: application to shallow flows. Comput. Methods Appl. Mech. Engrg., 195(19-22):2788-2815, 2006.

[8] F. H. Chu, W. D. Pilkey, and O. H. Pilkey. An analytical study of turbidity current steady flow. Marine Geology, 33(3-4):205-220, 1979. Cited By (since 1996): 11.

[9] G. Dal Maso, P. G. Lefloch, and F. Murat. Definition and weak stability of nonconservative products. J. Math. Pures Appl. (9), 74(6):483-548, 1995.

[10] W. Dietrich. Settling velocities of natural particles. Water Resources Research, 18(6):1615-1626, 1982.

[11] M. Garcia and G. Parker. Experiments on the entrainment of sediment into suspension by a dense bottom current. Journal of Geophysical Research, 98(C3):4793-4808, 1993.

[12] M. H. García. Hydraulic jumps in sediment-driven bottom currents. Journal of Hydraulic Engineering, 119(10):1094-1117, 1993.

[13] A. Grass. Sediment transport by waves and currents. SERC London Cent. Mar. Technol, Report No. FL29, 1981.

[14] S. M. Khan, J. Imran, S. Bradford, and J. Syvitski. Numerical modeling of hyperpycnal plume. Marine Geology, 222-223:193-211, November 2005.

[15] B. Kneller and C. Buckee. The structure and fluid mechanics of turbidity currents: a review of some recent studies and their geological implications. Sedimentology, 47:62-94, Feb. 2000.

[16] Y. Kubo. Experimental and numerical study of topographic effects on deposition from twodimensional, particle-driven density currents. Sedimentary Geology, 164(3-4):311-326, February 2004.

[17] Y. Kubo and T. Nakajima. Laboratory experiments and numerical simulation of sediment-wave formation by turbidity currents. Marine Geology, 192(1-3):105-121, December 2002.

[18] E. Meyer-Peter and R. Müller. Formulas for bed-load transport. In 2nd meeting IAHSR, Stockholm, Sweden, pages 1-26, 1948. 
[19] G. V. Middleton. Experiments on density and turbidity currents: Iii. deposition of sediment. Canadian Journal of Earth Sciences, 4:297-307, 1967.

[20] P. Nielsen. Coastal Bottom Boundary Layers and Sediment Transport. World Scientific Pub Co Inc, new ed edition, Aug. 1992.

[21] C. Parés. Numerical methods for nonconservative hyperbolic systems: a theoretical framework. SIAM J. Numer. Anal., 44(1):300-321 (electronic), 2006.

[22] C. Parés and M. Castro. On the well-balance property of Roe's method for nonconservative hyperbolic systems. Applications to shallow-water systems. M2AN Math. Model. Numer. Anal., $38(5): 821-852,2004$.

[23] G. Parker, Y. Fukushima, and H. M. Pantin. Self-accelerating turbidity currents. Journal of Fluid Mechanics, 171:145-181, 1986.

[24] I. Toumi. A weak formulation of Roe's approximate Riemann solver. J. Comput. Phys., 102(2):360-373, 1992. 